\title{
Majorana Representations of the Symmetric Group of Degree 4
}

\author{
A.A. Ivanov, D.V. Pasechnik, Á. Seress, S. Shpectorov
}

June 12, 2010 


\begin{abstract}
The Monster group $M$ acts on a real vector space $V_{M}$ of dimension 196,884 which is the sum of a trivial 1-dimensional module and a minimal faithful $M$-module. There is an $M$-invariant scalar product ( , ) on $V_{M}$, an $M$-invariant bilinear commutative non-associative algebra product - on $V_{M}$ (commonly known as the Conway-GriessNorton algebra), and a subset $A$ of $V_{M} \backslash\{0\}$ indexed by the $2 A$-involutions in $M$. Certain properties of the quintet

$$
\mathcal{M}=\left(M, V_{M}, A,(,), \cdot\right)
$$

have been axiomatized in Chapter 8 of [Iv09] under the name of Majorana representation of $M$. The axiomatization enables one to study Majorana representations of an arbitrary group $G$ (generated by its involutions). A representation might or might not exist, but it always exists whenever $G$ is a subgroup in $M$ generated by the $2 A$ involutions contained in $G$. We say that thus obtained representation is based on an embedding of $G$ in the Monster. The essential motivation for introducing the Majorana terminology was the most remarkable result by S. Sakuma [Sak07] which gave a classification of the Majorana representations of the dihedral groups. There are nine such representations and every single one is based on an embedding in the Monster of the relevant dihedral group. It is a well known fundamental property of the Monster that its 2A-involutions form a class of 6 -transpositions and that there are precisely nine $M$-orbits on the pairs of $2 A$-involutions (and also on the set of $2 A$-generated dihedral subgroups in $M$ ). In the present paper we are making a further step in building up the Majorana theory by classifying the Majorana representations of the symmetric group $S_{4}$ of degree 4 . We prove that $S_{4}$ possesses precisely four Majorana representations. The Monster is known to contain four classes of $2 A$-generated $S_{4}$-subgroups, so each of the four representations is based on an embedding of $S_{4}$ in the Monster. The classification of $2 A$-generated $S_{4}$-subgroups in the Monster relies on calculations with the character table of the Monster. Our elementary treatment shows that there are (at most) four isomorphism types of subalgebras in the Conway-Griess-Norton algebra of the Monster generated by six Majorana axial vectors canonically indexed by the transpositions of $S_{4}$. Two of these subalgebras are 13-dimensional, the other two have dimensions 9 and 6 . These dimensions, not to mention the isomorphism type of the subalgebras, were not known before.
\end{abstract}




\section{Internal Majorana representations}

We start with a version of the definition introduced in Section 8.6 in [Iv09]. Let $V$ be a real vector space equipped with a positive definite symmetric bilinear form $($, and a bilinear commutative non-associative algebra product $\cdot$. Suppose that

(M1) $($,$) associates with \cdot$ in the sense that

$$
(u, v \cdot w)=(u \cdot v, w)
$$

for all $u, v, w \in V$;

(M2) the Norton inequality holds, so that

$$
(u \cdot u, v \cdot v) \geq(u \cdot v, u \cdot v)
$$

for all $u, v \in V$.

Let $A$ be a subset of $V \backslash\{0\}$ and suppose that for every $a \in A$ the following conditions (M3) to (M7) hold.

(M3) $(a, a)=1$ and $a \cdot a=a$, so that the elements of $A$ are idempotents of length 1 ;

(M4) $V=V_{1}^{(a)} \oplus V_{0}^{(a)} \oplus V_{\frac{1}{2^{2}}}^{(a)} \oplus V_{\frac{1}{2^{5}}}^{(a)}$, where $V_{\mu}^{(a)}=\{v \mid v \in V, a \cdot v=\mu v\}$ is the set of $\mu$-eigenvectors of (the adjoint action of) $a$ on $V$;

(M5) $V_{1}^{(a)}=\{\lambda a \mid \lambda \in \mathbf{R}\}$

(M6) the linear transformation $\tau(a)$ of $V$ defined via

$$
\tau(a): u \mapsto(-1)^{2^{5} \mu} u
$$

for $u \in V_{\mu}^{(a)}$ with $\mu=1,0, \frac{1}{2^{2}}, \frac{1}{2^{5}}$, preserves the algebra product $\left(i . e . u^{\tau(a)} \cdot v^{\tau(a)}=\right.$ $(u \cdot v)^{\tau(a)}$ for all $\left.u, v \in V\right)$;

$(\mathrm{M} 7)$ if $V_{+}^{(a)}$ is the centralizer of $\tau(a)$ in $V$, so that $V_{+}^{(a)}=V_{1}^{(a)} \oplus V_{0}^{(a)} \oplus V_{\frac{1}{2^{2}}}^{(a)}$, then the linear transformation $\sigma(a)$ of $V_{+}^{(a)}$ defined via

$$
\sigma(a): u \mapsto(-1)^{2^{2} \mu} u
$$

for $u \in V_{\mu}^{(a)}$ with $\mu=1,0, \frac{1}{2^{2}}$ preserves the restriction of the algebra product to $V_{+}^{(a)}$ (i.e. $u^{\sigma(a)} \cdot v^{\sigma(a)}=(u \cdot v)^{\sigma(a)}$ for all $\left.u, v \in V_{+}^{(a)}\right)$. 
The elements of $A$ are called Majorana axes while the automorphisms $\tau(a)$ are called Majorana involutions.

Let $B$ be a set of Majorana axes, let $G$ be the subgroup in $G L(V)$ generated by the Majorana involutions $\tau(a)$ taken for all $a \in B$, let $U$ be the subalgebra in $(V, \cdot)$ generated by $B$, let $\left.()\right|_{U$,$} and \left.\cdot\right|_{U}$ be the restrictions to $U$ of $($,$) and \cdot$, respectively. Then the quintet

$$
\left(G, U, B,\left.(,)\right|_{U},\left.\cdot\right|_{U}\right)
$$

is said to be an internal Majorana representation of $G$. When studying Majorana representations of a group $G$, the whole of $V$ and $A$ are rather irrelevant, although $G$ might act unfaithfully, or even trivially on $U$, thus $V$ is needed mostly implicitly to make certain which group we are representing.

The major result of this paper is the following.

Theorem 1.1. The group $G=S_{4}$ has exactly four Majorana representations. All four representations are based on an embedding of $G$ in the Monster.

In the next few lemmas we state some frequently used consequences of the above definitions (where $a$ is a Majorana axis). The first two are the standard consequences of (M1).

Lemma 1.2. The decomposition in (M3) is ( , )-orthogonal so that $(u, v)=0$ whenever $u \in V_{\mu}^{(a)}, v \in V_{\lambda}^{(a)}$ and $\mu \neq \lambda$.

Lemma 1.3. Whenever $u, v \in V_{0}^{(a)}$, the equality $(u \cdot v, a)=0$ holds.

The next lemma is a consequence of (M3), (M5), and Lemma 1.2.

Lemma 1.4. For every $v \in V$ and $a \in A$ the vector $(a, v) a$ is the projection of $v$ to $V_{1}^{(a)}$.

Let $S p=\left\{1,0, \frac{1}{2^{2}}, \frac{1}{2^{5}}\right\}$ be the spectrum of the adjoint action on $a$ on $V$, so that

$$
V=\bigoplus_{\nu \in S p} V_{\nu}^{(a)} .
$$

Lemma 1.5. The eigenspaces of a Majorana axis satisfy the fusion rules described by Table 1 (whose rows and columns are indexed by the elements of Sp and whose entries are subsets of $S p$ ) 
Table 1

\begin{tabular}{|c|cccc|}
\hline & 1 & 0 & $\frac{1}{2^{2}}$ & $\frac{1}{2^{5}}$ \\
\hline 1 & 1 & 0 & $\frac{1}{2^{2}}$ & $\frac{1}{2^{5}}$ \\
0 & 0 & 0 & $\frac{1}{2^{2}}$ & $\frac{1}{2^{5}}$ \\
$\frac{1}{2^{2}}$ & $\frac{1}{2^{2}}$ & $\frac{1}{2^{2}}$ & 1,0 & $\frac{1}{2^{5}}$ \\
$\frac{1}{2^{5}}$ & $\frac{1}{2^{5}}$ & $\frac{1}{2^{5}}$ & $\frac{1}{2^{5}}$ & $1,0, \frac{1}{2^{2}}$ \\
\hline
\end{tabular}

which means that

$$
V_{\lambda}^{(a)} \cdot V_{\mu}^{(a)} \subseteq \bigoplus_{\nu \in S(\lambda, \mu)} V_{\nu}^{(a)}
$$

where $\lambda, \mu \in S p$ and $S(\mu, \lambda)$ is the $(\lambda, \mu)$-entry in Table 1 .

Proof. Table 1 follows directly from (M6) and (M7) together with Lemma 1.3 (the latter excludes the possibility for the eigenvalue 1 to appear in the $(0,0)$-th entry).

The assertion in the above lemma is easily seen to be equivalent to (M6) and (M7) (provided that (M2) holds). A few specific features of the fusion rules deserve a special attention.

Lemma 1.6. If $\alpha_{1}, \alpha_{2} \in V_{0}^{(a)}$ and $\beta_{1}, \beta_{2} \in V_{\frac{1}{2^{2}}}^{(a)}$ for a Majorana axis a then

$$
a \cdot\left(\alpha_{1} \cdot \alpha_{2}\right)=0, \quad a \cdot\left(\beta_{1} \cdot \beta_{2}-\left(\beta_{1} \cdot \beta_{2}, a\right) a\right)=0
$$

and

$$
a \cdot\left(\alpha_{1} \cdot \beta_{1}\right)=\frac{1}{2^{2}}\left(\alpha_{1} \cdot \beta_{1}\right)
$$

The following lemma whose rudiments can already be found in [Sak07] has matured under the name of the resurrection principle.

Lemma 1.7. Let $\left(G, U, B,\left.()\right|_{U},,\left.\cdot\right|_{U}\right)$ be a Majorana representation of a group $G$, let $W$ be a subspace of $U$, and let

$$
S=\left\{a, s, x, w_{\alpha}, w_{\beta}\right\}
$$


be a subset of $W$, where $a$ is a Majorana axis. Suppose that $W$ contains the products $\zeta \cdot \eta$ and $a \cdot(\zeta \cdot \eta)$ for all $\zeta, \eta \in S$ except possibly

$$
s \cdot s, \quad a \cdot(s \cdot s), \text { and } a \cdot(x \cdot x) .
$$

Then the latter three products are also contained in $W$ whenever there is a 0eigenvector

$$
\alpha_{s}=s+\mu x+w_{\alpha}
$$

and $a \frac{1}{2^{2}}$-eigenvector

$$
\beta_{s}=s+\nu x+w_{\beta}
$$

of a for some $\mu, \nu \in \mathbf{R}$, where $\mu^{2}$ and $\nu^{2}$ are distinct and non-zero.

Proof. By Lemma 1.6,

$$
\varphi:=\alpha_{s} \cdot \alpha_{s}-\beta_{s} \cdot \beta_{s}+\left(\beta_{s} \cdot \beta_{s}, a\right) a
$$

is a 0 -eigenvector of $a$ and by the hypothesis $\varphi$ is expressible as a quadratic polynomial of vectors from $S$. This polynomial does not involve $s \cdot s$. Furthermore, the product of $a$ with all terms in $\varphi$ is in $W$, with the possible exception of $(x \cdot x) \cdot a$. Since $x \cdot x$ appears with the non-zero coefficient $\mu^{2}-\nu^{2}$ in $\varphi,(x \cdot x) \cdot a$ can be expressed as an element of $W$ from the equation $a \cdot \varphi=0$.

Similarly,

$$
\psi:=\alpha_{s} \cdot \alpha_{s}-\alpha_{s} \cdot \beta_{s}
$$

does not involve $s \cdot s$, and by the previous paragraph $\psi \cdot a \in W$. On the other hand, by Lemma 1.6 we have

$$
a \cdot \psi=a \cdot\left(\alpha_{s} \cdot \alpha_{s}\right)-a \cdot\left(\alpha_{s} \cdot \beta_{s}\right)=0-\frac{1}{2^{2}}\left(\alpha_{s} \cdot \beta_{s}\right) .
$$

Since $s \cdot s$ appears in $\alpha_{s} \cdot \beta_{s}$ as a linear term with a non-zero coefficient, it is expressible as a linear combination of vectors in $W$. Finally, from $\left(\alpha_{s} \cdot \alpha_{s}\right) \cdot a=0$ we can express $(s \cdot s) \cdot a$ as an element of $W$.

Although $s \cdot s$ has been eliminated in $\alpha_{s} \cdot \alpha_{s}-\alpha_{s} \cdot \beta_{s}$, it resurrects (only quartered) after multiplying the expression by $a$ (hence the name of the principle). Note that the proof of Lemma 1.7 also shows that if expressions for the products $\zeta \cdot \eta$ and $a \cdot(\zeta \cdot \eta)$ are known as linear combinations of some generating set $\mathcal{B}$ of $W$ then we can compute $s \cdot s, a \cdot(s \cdot s)$, and $a \cdot(x \cdot x)$ as linear combinations of $\mathcal{B}$ as well.

The following junior version of the resurrection principle will be commonly used. A subspace $W$ of $U$ will be said to be $a$-stable if it contains the product $a \cdot w$ for every $w \in W$. 
Lemma 1.8. Let $\left(G, U, B,\left.()\right|_{U},,\left.\cdot\right|_{U}\right)$ be a Majorana representation of a group $G$. Let a be a Majorana axis, and let $W$ be an a-stable subspace of $U$. For $s \in U$ suppose that

$$
\alpha_{s}=s+w_{\alpha} \text { and } \beta_{s}=s+w_{\beta}
$$

are 0 - and $\frac{1}{2^{2}}$-eigenvectors of $a$, respectively, for some $w_{\alpha}, w_{\beta} \in W$. Then

$$
s=-\left[4 a \cdot\left(w_{\alpha}-w_{\beta}\right)+w_{\beta}\right]
$$

in particular $s \in W$.

Proof. We have

$$
a \cdot\left(w_{\alpha}-w_{\beta}\right)=a \cdot\left(\alpha_{s}-\beta_{s}\right)=0-\frac{1}{2^{2}} \beta_{s}=-\frac{1}{2^{2}} s-\frac{1}{2^{2}} w_{\beta}
$$

and the assertion is immediate.

The following result is well known and crucial both in the Vertex Operator Algebra (Lemma 9.1 in [Miy04]) and the Monster (Section 13 in [C84]) contexts.

Lemma 1.9. If $a$ and $b$ are distinct Majorana axes then

$$
0 \leq(a, b) \leq 1 / 3
$$

The following pretty lemma proved to be very useful. It expands a lemma on p. 532 in [C84] and Proposition 6.9 in [Miy96]) (both heavily based on the Norton inequality) from the Monster algebra to an arbitrary Majorana representation. Recall that $v$ and $u$ from $V$ are said to associate if

$$
v \cdot(w \cdot u)=(v \cdot w) \cdot u
$$

for every $w \in V$.

Lemma 1.10. A Majorana axis associates with every element of its 0-eigenspace.

Proof. Let $a$ be a Majorana axis, and let $\alpha$ be a 0-eigenvector for $a$. Then for a $\mu$-eigenvector $v$ of $a$, on one hand we have

$$
(a \cdot v) \cdot \alpha=(\mu v) \cdot \alpha=\mu(v \cdot \alpha) .
$$

On the other hand, by the fusion rules, $v \cdot \alpha$ is also a $\mu$-eigenvector for $a$ and so

$$
a \cdot(v \cdot \alpha)=\mu(v \cdot \alpha) .
$$

So, by distributivity, $a$ and $\alpha$ associate.

If $a_{0}$ and $a_{1}$ are two Majorana axis generating a $2 B$-algebra (cf. Table 3), then applying Lemma 1.10 for $a=a_{0}$ and $\alpha=a_{1}$, we conclude that $a_{0}$ and $a_{1}$ associate. 


\section{An explicit version of Sakuma's theorem}

In this section we revisit Sakuma's classification of the Majorana representations of the dihedral groups aiming

(a) to extract the classification from the Vertex Operator Algebra context it was originally placed in;

(b) to make the computational core of the proof more transparent by using the resurrection principle explicitly;

(c) to carry on the classification beyond the upper bound on the number of representations in order to make the existence part Monster-independent;

(d) to present the proof with inner and algebra products scaled suitably for our future purposes.

A further aim has emerged within the process of revising the proof:

(e) to show that (in the case of algebras generated by a pair of Majorana axes) the Norton inequality is a consequence of the remaining conditions.

We give a short version of Sakuma's result as follows. For a more detailed description, see Subsection 2.6.

Theorem 2.1. Dihedral groups have exactly nine Majorana representations. All nine representations are based on an embedding in the Monster.

The scaling in [Sak07] was dictated by the Vertex Operator Algebra environment, particularly the Majorana axes correspond to conformal vectors of central charge $\frac{1}{2}$ (so that they are doubled idempotents). In the Monster context [C84], [N96], [ATLAS] the scaling is inherited from the construction of the Monster in terms of the Leech vectors.

Suppose that $(V, A,(),, \cdot)$ is a quadruple satisfying (M1) to (M7). Let $a_{0}, a_{1} \in A$ be a pair of Majorana axes, let $\tau_{0}=\tau\left(a_{0}\right), \tau_{1}=\tau\left(a_{1}\right)$ be the corresponding Majorana involutions, let $D$ be the subgroup in $G L(V)$ generated by $\tau_{0}$ and $\tau_{1}$, and let $U$ be the subalgebra in $(V, \cdot)$ generated by $a_{0}$ and $a_{1}$, so that

$$
\left(D, U,\left\{a_{0}, a_{1}\right\},\left.(,)\right|_{U},\left.\cdot\right|_{U}\right)
$$

is a Majorana representation of $D$. Our exposition is divided into a number of steps dealt with in individual subsections. 


\subsection{Symmetric generating set}

We start by producing a $D$-invariant generating set $B$ of $U$. Let $\rho=\tau_{0} \tau_{1}$ be the generator of the rotation group of $D$. For an integer $i$ and $\varepsilon \in\{0,1\}$ let $a_{2 i+\varepsilon}$ denote the images of $a_{\varepsilon}$ under the $i$-th power of $\rho$. Notice that $a_{-1}$ is the image of $a_{1}$ under $\tau_{0}$. Let

$$
B_{\varepsilon}=\left\{a_{2 i+\varepsilon} \mid i \in \mathbf{Z}\right\} \text { and } B=B_{0} \cup B_{1} .
$$

Then $a_{2 i+\varepsilon}$ is a Majorana axis and the corresponding Majorana involution

$$
\tau\left(a_{2 i+\varepsilon}\right)=\rho^{-i} \tau_{\varepsilon} \rho^{i}
$$

will be denoted by $\tau_{2 i+\varepsilon}$.

Lemma 2.2. The set $B$ is $D$-invariant and $B$ is contained in the subalgebra $U$.

Proof. The $D$-invariance is rather obvious. By (M4) the vector $a_{1}$ possesses a unique presentation of the form

$$
a_{1}=\lambda_{1} a_{0}+\alpha_{1}+\beta_{1}+\gamma_{1}
$$

where $\lambda_{1}=\left(a_{0}, a_{1}\right), a_{0} \cdot \alpha_{1}=0, a_{0} \cdot \beta_{1}=\frac{1}{2^{2}} \beta_{1}$ and $a_{0} \cdot \gamma_{1}=\frac{1}{2^{5}} \gamma_{1}$. Considering the relevant Vandermode matrix one can express the eigenvectors $\alpha_{1}, \beta_{1}$ and $\gamma_{1}$ as linear combinations of the vectors

$$
a_{0}, \quad a_{1}, \quad a_{0} \cdot a_{1} \text {, and } a_{0} \cdot\left(a_{0} \cdot a_{1}\right) .
$$

Thus $U$ contains $\alpha_{1}, \beta_{1}$ and $\gamma_{1}$. Since $a_{-1}$ is the image of $a_{1}$ under $\tau_{0}$, by (M6) we have

$$
a_{-1}=\lambda_{1} a_{0}+\alpha_{1}+\beta_{1}-\gamma_{1},
$$

so that

$$
\gamma_{1}=\frac{1}{2}\left(a_{1}-a_{-1}\right)
$$

Since $U$ is already known to contain $a_{1}$ and $\gamma_{1}$, it also contains $a_{-1}$ and the proof is easy to accomplish arguing by induction.

\subsection{Multiplying two symmetric generators}

A particular vector denoted by $\sigma_{1}$ plays the most important role in the subsequent development.

Lemma 2.3. The vector

$$
\sigma_{1}=a_{0} \cdot a_{1}-\frac{1}{2^{5}}\left(a_{0}+a_{1}\right)
$$

is D-invariant. 
Proof. Since

$$
a_{0} \cdot a_{1}=\lambda_{1} a_{0}+\frac{1}{2^{2}} \beta_{1}+\frac{1}{2^{5}} \gamma_{1}
$$

while

$$
a_{1}=\lambda_{1} a_{0}+\alpha_{1}+\beta_{1}+\gamma_{1}
$$

the difference $a_{0} \cdot a_{1}-\frac{1}{2^{5}} a_{1}$ is contained in $V_{+}^{\left(a_{0}\right)}=C_{V}\left(\tau_{0}\right)$ and hence it is centralized by $\tau_{0}$. Since $a_{0}$ is also centralized by $\tau_{0}$, and in view of the symmetry between $a_{0}$ and $a_{1}$, the vector $\sigma_{1}$ is contained in $V_{+}^{\left(a_{0}\right)} \cap V_{+}^{\left(a_{1}\right)} \leq C_{V}(D)$.

Lemma 2.4. The following equalities hold:

$$
\begin{array}{r}
\alpha_{1}=-4 \sigma_{1}+\left(3 \lambda_{1}-\frac{1}{2^{3}}\right) a_{0}+\frac{7}{2^{4}}\left(a_{1}+a_{-1}\right), \\
\beta_{1}=4 \sigma_{1}-\left(4 \lambda_{1}-\frac{1}{2^{3}}\right) a_{0}+\frac{1}{2^{4}}\left(a_{1}+a_{-1}\right), \\
a_{j} \cdot \sigma_{1}=\frac{7}{2^{5}} \sigma_{1}+\left(\frac{3 \lambda_{1}}{2^{2}}-\frac{25}{2^{10}}\right) a_{j}+\frac{7}{2^{11}}\left(a_{j-1}+a_{j+1}\right), \\
\sigma_{1}=\left(\frac{31 \lambda_{1}}{2^{5}}-\frac{1}{2^{5}}\right) a_{0}-\frac{1}{2^{5}} \alpha_{1}+\frac{7}{2^{5}} \beta_{1} .
\end{array}
$$

Proof. The first two equalities are obtained by expressing $\alpha_{1}$ and $\beta_{1}$ from

$$
a_{1}=\lambda_{1} a_{0}+\alpha_{1}+\beta_{1}+\frac{1}{2}\left(a_{1}-a_{-1}\right)
$$

and

$$
a_{0} \cdot a_{1}=\lambda_{1} a_{0}+\frac{1}{2^{2}} \beta_{1}+\frac{1}{2^{6}}\left(a_{1}-a_{-1}\right)
$$

followed by substituting $\sigma_{1}+\frac{1}{2^{5}}\left(a_{0}+a_{1}\right)$ in the place of $a_{0} \cdot a_{1}$. For the case $j=0$ the third equality can be deduced by multiplying the second one by $a_{0}$, and using Lemma 2.3 to express $a_{0} \cdot a_{1}$ and $a_{0} \cdot a_{-1}$ as linear combinations of $\sigma_{1}, a_{0}, a_{1}$, and $a_{-1}$. If $j=1$ then the equality holds because of the symmetry between $a_{0}$ and $a_{1}$. The generic case follows from the $D$-invariance of $\sigma_{1}$.

By analogy, one can introduce the product of $a_{i}$ and $a_{j}$ shifted by $-\frac{1}{2^{5}}\left(a_{i}+a_{j}\right)$ to obtain a vector invariant under the subgroup in $D$ generated by $\tau_{i}$ and $\tau_{j}$. We will deal with two such vectors attaining the full $D$-invariance.

Lemma 2.5. Each of the vectors

$$
\sigma_{2,1}=a_{1} \cdot a_{-1}-\frac{1}{2^{5}}\left(a_{1}+a_{-1}\right) \text { and } \sigma_{2,0}=a_{0} \cdot a_{2}-\frac{1}{2^{5}}\left(a_{0}+a_{2}\right)
$$

is D-invariant. 
Proof. Arguing as in the proof of Lemma 2.3, we obtain the $\tau_{1}$-invariance of $\sigma_{2,1}$ and the $\tau_{0}$-invariance of $\sigma_{2,0}$. Since $\tau_{0}$ permutes $a_{1}$ with $a_{-1}$ while $\tau_{1}$ permutes $a_{0}$ with $a_{2}$, the full invariance follows.

\subsection{Angles between symmetric generators}

For $\varepsilon \in\{0,1\}$ we define $\lambda_{j}^{(\varepsilon)}=\left(a_{\varepsilon}, a_{j+\varepsilon}\right)$. Then we have the following lemma, which is Proposition 3.1 in [Sak07].

Lemma 2.6. For any two integers $k$ and $j$ the inner product $\left(a_{k}, a_{k+j}\right)$ is uniquely determined by $j$. In particular, $\lambda_{j}^{(\varepsilon)}$ does not depend on $\varepsilon$.

Proof. Since $D$ acts transitively both on $B_{0}$ and on $B_{1}$ preserving the inner products, all we need is to show that $\lambda_{j}^{(0)}=\lambda_{j}^{(1)}$ for all $j$. Also by the $D$-transitivity $\lambda_{j}^{(0)}=\lambda_{j}^{(1)}$ whenever $j$ is odd. Since $\tau_{0}$ permutes $a_{j}$ and $a_{-j}$, we can assume without loss that $j$ is positive and argue by induction, since we already know that $\lambda_{1}^{(0)}=\lambda_{1}^{(1)}=\lambda_{1}$ and that $\lambda_{0}^{(0)}=\lambda_{0}^{(1)}=1$ by (M3). Thus we assume that for all $0 \leq k \leq j$ the value of $\lambda_{k}^{(0)}$ equals to that of $\lambda_{k}^{(1)}$ and is denoted by $\lambda_{k}$. Equation (3) in Lemma 2.4 for $j=0$ and $j=1$ can be rewritten as follows:

$$
\begin{gathered}
\frac{7}{2^{11}}\left(a_{1}+a_{-1}\right)=a_{0} \cdot \sigma_{1}-\frac{7}{2^{5}} \sigma_{1}-\left(\frac{3 \lambda_{1}}{2^{2}}-\frac{25}{2^{10}}\right) a_{0}, \\
\frac{7}{2^{11}}\left(a_{0}+a_{2}\right)=a_{1} \cdot \sigma_{1}-\frac{7}{2^{5}} \sigma_{1}-\left(\frac{3 \lambda_{1}}{2^{2}}-\frac{25}{2^{10}}\right) a_{1} .
\end{gathered}
$$

Evaluating the inner product of both sides of the former equality with $a_{j}$ and of the latter one with $a_{j+1}$ we obtain

$$
\begin{gathered}
\frac{7}{2^{11}}\left(\lambda_{j-1}+\lambda_{j+1}^{(1)}\right)=\left(a_{j}, a_{0} \cdot \sigma_{1}\right)-\frac{7}{2^{5}}\left(a_{j}, \sigma_{1}\right)-\left(\frac{3 \lambda_{1}}{2^{2}}-\frac{25}{2^{10}}\right) \lambda_{j}, \\
\frac{7}{2^{11}}\left(\lambda_{j+1}^{(0)}+\lambda_{j-1}\right)=\left(a_{j+1}, a_{1} \cdot \sigma_{1}\right)-\frac{7}{2^{5}}\left(a_{j+1}, \sigma_{1}\right)-\left(\frac{3 \lambda_{1}}{2^{2}}-\frac{25}{2^{10}}\right) \lambda_{j} .
\end{gathered}
$$

By (M1), $\left(a_{j}, a_{0} \cdot \sigma_{1}\right)=\left(a_{j} \cdot a_{0}, \sigma_{1}\right)$ and $\left(a_{j+1}, a_{1} \cdot \sigma_{1}\right)=\left(a_{j+1} \cdot a_{1}, \sigma_{1}\right)$. Since $\lambda_{j+1}^{(0)}=\lambda_{j+1}^{(1)}$ whenever $j+1$ is odd, we may assume without loss that $j$ is odd, in which case $D$ contains an element which maps the pair $\left\{a_{0}, a_{j}\right\}$ onto the pair $\left\{a_{1}, a_{j+1}\right\}$. Thus all four inner products in the penultimate sentence are equal. Similarly, there is an element of $D$ which maps $\left\{a_{j}, a_{j+1}\right\}$ onto $\left\{a_{0}, a_{1}\right\}$, and $\left(a_{0}, \sigma_{1}\right)=\left(a_{1}, \sigma_{1}\right)$ because of the symmetry between $a_{0}$ and $a_{1}$ in the defining formula of $\sigma_{1}$. Hence the inductive step follows. 
Since $a_{k}=a_{j}$ if and only if $\left(a_{k}, a_{j}\right)=1$, from the above lemma we obtain directly the following important consequence.

Lemma 2.7. The sets $B_{0}$ and $B_{1}$ contain the same number of vectors.

We conclude this subsection by the following.

Lemma 2.8. The following equalities hold:

$$
\begin{array}{r}
a_{2 i} \cdot \sigma_{2,0}=\frac{7}{2^{5}} \sigma_{2,0}+\left(\frac{3 \lambda_{2}}{2^{2}}-\frac{25}{2^{10}}\right) a_{2 i}+\frac{7}{2^{11}}\left(a_{2 i-2}+a_{2 i+2}\right), \\
a_{2 i+1} \cdot \sigma_{2,1}=\frac{7}{2^{5}} \sigma_{2,1}+\left(\frac{3 \lambda_{2}}{2^{2}}-\frac{25}{2^{10}}\right) a_{2 i+1}+\frac{7}{2^{11}}\left(a_{2 i-1}+a_{2 i+3}\right) .
\end{array}
$$

Proof. A proof can be achieved as a minor generalization of that for equation (3) in Lemma 2.4, making use of the $D$-invariance established in Lemma 2.5 and the equality $\lambda_{2}^{(0)}=\lambda_{2}^{(1)}$ proved in Lemma 2.6.

The following useful lemma did not appear explicitly in [Sak07], but it clearly belongs to this subsection.

Lemma 2.9. If $B_{0}=B_{1}$ then for any two non-zero integers $j$ and $k$ we have $\lambda_{j}=\lambda_{k}$.

Proof. The form ( , ) associates with the algebra product, which gives

$$
\left(a_{0} \cdot a_{j}, a_{k}\right)=\left(a_{j}, a_{0} \cdot a_{k}\right)
$$

Since $B_{0}=B_{1}$, we have $a_{i} \cdot a_{i+j}=\sigma_{j}+\frac{1}{2^{5}}\left(a_{i}+a_{i+j}\right)$. Taking the inner product of the two sides of this equation with $a_{i}$ gives $\left(a_{i}, \sigma_{j}\right)=\frac{31 \lambda_{j}}{2^{5}}-\frac{1}{2^{5}}$ for all values of $i$. Thus (multiplying by 32 to eliminate the fractions) we obtain

$$
31 \lambda_{j}+\lambda_{k}+\lambda_{j-k}=31 \lambda_{k}+\lambda_{j}+\lambda_{j-k}
$$

and the claim follows.

\subsection{Bounding the dimension}

Let $W$ be the subspace in $U$ spanned by the set

$$
X=\left\{a_{0}, a_{1}, a_{-1}, a_{2}, a_{-2}, \sigma_{1}, \sigma_{2,0}, \sigma_{2,1}\right\}
$$

so that the dimension of $W$ is at most eight. We are going to show that $W$ is in fact the whole of $U$ and start with the following. 
Lemma 2.10. The subspace $W$ contains $\sigma_{1} \cdot \sigma_{1}, a_{0} \cdot\left(\sigma_{1} \cdot \sigma_{1}\right)$ and $a_{0} \cdot \sigma_{2,1}$.

Proof. We apply the resurrection principle Lemma 1.7 with

$$
s=\sigma_{1}, x=a_{1}+a_{-1}, \alpha_{s}=-\frac{1}{2^{2}} \alpha_{1}, \beta_{s}=\frac{1}{2^{2}} \beta_{1},
$$

in which case $w_{\alpha}$ and $w_{\beta}$ are multiples of $a_{0}$, while

$$
x \cdot x=2 \sigma_{2,1}+\frac{17}{2^{4}}\left(a_{1}+a_{-1}\right) .
$$

Then the hypothesis of Lemma 1.7 holds by Lemmas 2.4 and 2.5.

The explicit formulas related to Lemma 2.10, as calculated in [GAP4], are the following: 


$$
\begin{aligned}
& \alpha_{1} \cdot \alpha_{1}=16 \sigma_{1} \cdot \sigma_{1}-\frac{49}{2^{5}} \sigma_{1}+\frac{49}{2^{7}} \sigma_{2,1}+ \\
& \left(-9 \lambda_{1}^{2}+\frac{3}{2^{2}} \lambda_{1}-\frac{81}{2^{11}}\right) a_{0}+\left(-\frac{21}{2^{3}} \lambda_{1}+\frac{1183}{2^{12}}\right)\left(a_{1}+a_{-1}\right)-\frac{49}{2^{12}}\left(a_{2}+a_{-2}\right) \\
& \beta_{1} \cdot \beta_{1}=16 \sigma_{1} \cdot \sigma_{1}+\left(-8 \lambda_{1}+\frac{15}{2^{5}}\right) \sigma_{1}+\frac{1}{2^{7}} \sigma_{2,1}+ \\
& \left(-8 \lambda_{1}^{2}+\frac{1}{2} \lambda_{1}-\frac{9}{2^{11}}\right) a_{0}+\left(\frac{1}{2^{2}} \lambda_{1}-\frac{17}{2^{12}}\right)\left(a_{1}+a_{-1}\right)+\frac{7}{2^{12}}\left(a_{2}+a_{-2}\right) \\
& \alpha_{1} \cdot \beta_{1}=-16 \sigma_{1} \cdot \sigma_{1}+\left(3 \lambda_{1}+\frac{17}{2^{5}}\right) \sigma_{1}+\frac{7}{2^{7}} \sigma_{2,1}+ \\
& \left(9 \lambda_{1}^{2}-\frac{21}{2^{5}} \lambda_{1}+\frac{45}{2^{11}}\right) a_{0}+\left(\frac{75}{2^{6}} \lambda_{1}-\frac{39}{2^{12}}\right)\left(a_{1}+a_{-1}\right)+\frac{21}{2^{12}}\left(a_{2}+a_{-2}\right) \\
& \alpha_{1} \cdot \alpha_{1}-\beta_{1} \cdot \beta_{1}+\left(\beta_{1} \cdot \beta_{1}, a_{0}\right) a_{0}=\left(8 \lambda_{1}-2\right) \sigma_{1}+\frac{3}{2^{3}} \sigma_{2,1}+ \\
& \left(-2 \lambda_{1}^{2}+\frac{5}{2^{2}} \lambda_{1}+\frac{1}{2^{6}} \lambda_{2}-\frac{13}{2^{8}}\right) a_{0}+\left(-\frac{23}{2^{3}} \lambda_{1}+\frac{75}{2^{8}}\right)\left(a_{1}+a_{-1}\right)-\frac{7}{2^{9}}\left(a_{2}+a_{-2}\right)( \\
& a_{0} \cdot \sigma_{2,1}=-\frac{1}{3}\left[\left(-32 \lambda_{1}+\frac{19}{2^{4}}\right) \sigma_{1}-\frac{7}{2^{5}} \sigma_{2,0}+\right. \\
& \left.\left(32 \lambda_{1}^{2}-5 \lambda_{1}+\frac{1}{2^{3}} \lambda_{2}+\frac{127}{2^{10}}\right) a_{0}+\left(-\frac{1}{2} \lambda_{1}+\frac{19}{2^{10}}\right)\left(a_{1}+a_{-1}\right)-\frac{7}{2^{11}}\left(a_{2}+a_{-2}\right)\right] \\
& \alpha_{1} \cdot \beta_{1}+\beta_{1} \cdot \beta_{1}-\left(\beta_{1} \cdot \beta_{1}, a_{0}\right) a_{0}=\left(-5 \lambda_{1}+1\right) \sigma_{1}+\frac{1}{2^{4}} \sigma_{2,1}+ \\
& \left(2 \lambda_{1}^{2}-\frac{37}{2^{5}} \lambda_{1}-\frac{1}{2^{6}} \lambda_{2}+\frac{17}{2^{9}}\right) a_{0}+\left(\frac{91}{2^{6}} \lambda_{1}-\frac{7}{2^{9}}\right)\left(a_{1}+a_{-1}\right)+\frac{7}{2^{10}}\left(a_{2}+a_{-2}\right)(12 \\
& \sigma_{1} \cdot \sigma_{1}=\frac{1}{3}\left[\left(-\frac{5}{2^{2}} \lambda_{1}-\frac{13}{2^{9}}\right) \sigma_{1}-\frac{7}{2^{9}} \sigma_{2,0}+\frac{21}{2^{11}} \sigma_{2,1}\right]+ \\
& \frac{7}{3}\left[\left(\frac{1}{2} \lambda_{1}^{2}-\frac{1}{2^{7}} \lambda_{1}+\frac{1}{2^{9}} \lambda_{2}-\frac{1}{2^{15}}\right) a_{0}+\left(\frac{7}{2^{8}} \lambda_{1}-\frac{35}{2^{16}}\right)\left(a_{1}+a_{-1}\right)+\frac{7}{2^{16}}\left(a_{2}+a_{-2}\right)\right]
\end{aligned}
$$

Up to a rescaling, the last of the above formulas appeared in Proposition 3.2 of [Sak07].

Lemma 2.11. For every integer $j$ and $\varepsilon \equiv j \bmod 2$ the following equality holds:

$\sigma_{1} \cdot \sigma_{1}=\frac{1}{3}\left[\left(-\frac{5}{2^{2}} \lambda_{1}-\frac{13}{2^{9}}\right) \sigma_{1}-\frac{7}{2^{9}} \sigma_{2, \varepsilon}+\frac{21}{2^{11}} \sigma_{2, \varepsilon+1}\right]+$ 
$\frac{7}{3}\left[\left(\frac{1}{2} \lambda_{1}^{2}-\frac{1}{2^{7}} \lambda_{1}+\frac{1}{2^{9}} \lambda_{2}-\frac{1}{2^{15}}\right) a_{j}+\left(\frac{7}{2^{8}} \lambda_{1}-\frac{35}{2^{16}}\right)\left(a_{j+1}+a_{j-1}\right)+\frac{7}{2^{16}}\left(a_{j+2}+a_{j-2}\right)\right]$.

Proof. If $j=1$ then we take equation (13) and apply the complete symmetry between $a_{0}$ and $a_{1}$ in the definition of $\sigma_{1}$. Now the generic case follows from the $D$-invariance of $\sigma_{1}, \sigma_{2,0}$, and $\sigma_{2,1}$.

Lemma 2.12. The subspace $W$ contains the generating set $B$ of $U$ consisting of Majorana axes. Furthermore, the subspace $W$ is D-invariant.

Proof. We have to show that $W$ contains $a_{j}$ for every integer $j$. Equalizing the right hand sides of the equalities in Lemma 2.11 for $j=0$ and $j=1$, we can express $a_{3}$ as a linear combination of vectors in $X$ and then proceed by induction making use of Lemma 2.11. Thus the image of $X$ under every element of $D$ is contained in $W$ and the $D$-invariance follows.

Lemma 2.13. The subspace $W$ is a-stable for every $a \in B$.

Proof. We have to show that $a \cdot w \in W$ for every $a \in B$ and $w \in W$. Suppose first that $a=a_{0}$. We can assume without loss that $w \in X$ in which case the claim follows from the definitions of $\sigma_{1}$ and $\sigma_{2,0}$, equation (3) for $j=0$, equation (5) for $i=0$, and (11). The case $a=a_{1}$ now follows from the symmetry between $a_{0}$ and $a_{1}$ while the generic case is by the $D$-invariance of $W$ established in Lemma 2.12.

The next proposition is Lemma 3.5 in [Sak07].

Proposition 2.14. The algebra product · is closed on $W$.

Proof. We claim that $W$ is spanned by $B$ together with $\sigma_{1}$ and $\sigma_{2,0}$. This can be seen by equalizing the right hand sides of the equations in Lemma 2.11 for $j=0$ and $j=1$, and expressing $\sigma_{2,1}$ as a linear combination of $\sigma_{1}, \sigma_{2,0}$, and some axes from $B$ (this is possible since the $\sigma_{2,1}$-coefficients in the two equations are distinct constants). Thus, in view of Lemmas 2.10 and 2.13 it only remains to show that $W$ contains $\sigma_{2,0} \cdot \sigma_{2,0}$ and $\sigma_{1} \cdot \sigma_{2,0}$.

Consider the projections of $a_{2}$ onto the 0 - and $\frac{1}{2^{2}}$-eigenspaces of $a_{0}$ (compare to Lemma 2.4):

$$
\begin{gathered}
\alpha_{2}=-4 \sigma_{2,0}+\left(3 \lambda_{2}-\frac{1}{2^{3}}\right) a_{0}+\frac{7}{2^{4}}\left(a_{2}+a_{-2}\right), \\
\beta_{2}=4 \sigma_{2,0}-\left(4 \lambda_{2}-\frac{1}{2^{3}}\right) a_{0}+\frac{1}{2^{4}}\left(a_{2}+a_{-2}\right) .
\end{gathered}
$$

In view of Lemma 2.13, we achieve the goal applying Lemma 1.8 first for

$$
s=\sigma_{2,0} \cdot \sigma_{2,0}, \quad \alpha_{s}=\frac{1}{2^{4}} \alpha_{2} \cdot \alpha_{2}, \quad \beta_{s}=-\frac{1}{2^{4}} \alpha_{2} \cdot \beta_{2}
$$


and next for

$$
s=\sigma_{1} \cdot \sigma_{2,0}, \quad \alpha_{s}=\frac{1}{2^{4}} \alpha_{1} \cdot \alpha_{2}, \quad \beta_{s}=-\frac{1}{2^{4}} \alpha_{1} \cdot \beta_{2}
$$

This appears to be a good place to put the inner product values (calculated in [GAP4]) required to recover the form ( , ) on the whole of $W$.

Lemma 2.15. The following equalities hold for every $\varepsilon$ :

$$
\begin{gathered}
\lambda_{3}=\frac{1}{7}\left[-2^{15} \lambda_{1}^{3}+2^{12} \cdot 3^{2} \lambda_{1}^{2}-2^{7} \cdot 3 \cdot 5 \lambda_{1} \lambda_{2}-3^{2} \cdot 241 \lambda_{1}-3 \cdot 11 \lambda_{2}+3 \cdot 11\right] ; \\
\lambda_{4}=\frac{1}{7}\left[2^{23} \lambda_{1}^{4}-2^{15} \cdot 293 \lambda_{1}^{3}+2^{16} \cdot 7 \lambda_{1}^{2} \lambda_{2}+2^{12} \cdot 3^{3} \cdot 7 \lambda_{1}^{2}-2^{7} \cdot 5 \lambda_{1} \lambda_{2}-2^{7} \lambda_{2}^{2}-2^{7} \cdot 5 \cdot 31 \lambda_{1}-3 \cdot 7 \lambda_{2}+2^{2} \cdot 3 \cdot 13\right] ; \\
\left(\sigma_{1}, \sigma_{1}\right)=\frac{3}{2^{2}} \lambda_{1}^{2}+\frac{65}{2^{9}} \lambda_{1}+\frac{7}{2^{11}} \lambda_{2}-\frac{3}{2^{11}} ; \\
\left(\sigma_{1}, \sigma_{2, \varepsilon}\right)=-16 \lambda_{1}^{3}+18 \lambda_{1}^{2}-\frac{3}{2^{4}} \lambda_{1} \lambda_{2}-\frac{463}{2^{9}} \lambda_{1}-\frac{83}{2^{11}} \lambda_{2}+\frac{23}{2^{11}} ; \\
\left(\sigma_{2, \varepsilon}, \sigma_{2, \varepsilon}\right)=2^{12} \lambda_{1}^{4}-2^{4} \cdot 293 \lambda_{1}^{3}+2^{5} \cdot 7 \lambda_{1}^{2} \lambda_{2}+2 \cdot 3^{3} \cdot 7 \lambda_{1}^{2}-\frac{5}{2^{4}} \lambda_{1} \lambda_{2}+\frac{11}{2^{4}} \lambda_{2}^{2}-\frac{5 \cdot 31}{2^{4}} \lambda_{1}+\frac{239}{2^{11}} \lambda_{2}+\frac{3^{2} \cdot 17}{2^{11}} ; \\
\left(\sigma_{2, \varepsilon}, \sigma_{2, \varepsilon+1}\right)=2^{12} \lambda_{1}^{4}-\frac{2^{4} \cdot 11 \cdot 181}{7} \lambda_{1}^{3}+\frac{2^{5} \cdot 19}{7} \lambda_{1}^{2} \lambda_{2}+\frac{2 \cdot 3 \cdot 421}{7} \lambda_{1}^{2}+\frac{5 \cdot 389}{2^{4} \cdot 7} \lambda_{1} \lambda_{2}+\frac{17}{2^{4} \cdot 7} \lambda_{2}^{2} \\
-\frac{5 \cdot 17 \cdot 197}{2^{8} \cdot 7} \lambda_{1}-\frac{17 \cdot 191}{2^{11} \cdot 7} \lambda_{2}+\frac{3^{2} \cdot 17}{2^{11}} .
\end{gathered}
$$

Proof. These inner product values follow from the orthogonality of eigenvectors and the associative rule (M1). For example, the value $\left(\sigma_{1}, \sigma_{1}\right)$ can be computed from the orthogonality of $\alpha_{1}$ and $\beta_{1}$ given in (1) and (2). Knowing this value and using (13), we can solve $\left(\sigma_{1} \cdot \sigma_{1}, a_{0}-a_{1}\right)=\left(\sigma_{1}, \sigma_{1} \cdot\left(a_{0}-a_{1}\right)\right)$ for $\lambda_{3}$, etc.

\subsection{Bounding the gonality}

In this subsection (which corresponds to Section 4 of [Sak07]) we show that $B=$ $B_{0} \cup B_{1}$ contains at most six Majorana axes.

Lemma 2.16. If $|B| \geq 7$ then the vectors $a_{1}-a_{-1}, a_{2}-a_{-2}$ and $a_{3}-a_{-3}$ are linearly independent. 
Proof. Under the hypothesis of the lemma, by Lemma 2.5 the axes $a_{j}$ for $-3 \leq j \leq 3$ are pairwise distinct. Furthermore, in terms introduced in Subsection 2.3 we have

$$
\left(a_{i}-a_{-i}, a_{k}-a_{-k}\right)=2 \cdot\left(\lambda_{i-k}-\lambda_{i+k}\right) .
$$

for $1 \leq i, k \leq 3$. Since $\lambda_{0}=1$ and $\lambda_{j}=\lambda_{-j}$ for all $j$, the halved Gram matrix $M=\left\|\mu_{i k}\right\|_{3 \times 3}$ of the considered three vectors is the following:

$$
\left(\begin{array}{ccc}
1-\lambda_{2} & \lambda_{1}-\lambda_{3} & \lambda_{2}-\lambda_{4} \\
\lambda_{1}-\lambda_{3} & 1-\lambda_{4} & \lambda_{1}-\lambda_{5} \\
\lambda_{2}-\lambda_{4} & \lambda_{1}-\lambda_{5} & 1-\lambda_{6}
\end{array}\right) .
$$

We claim that $M$ is non-singular. By the school textbook formula

$$
\begin{aligned}
\operatorname{det}(M) & =\mu_{11} \mu_{22} \mu_{33}+\mu_{21} \mu_{32} \mu_{13}+\mu_{31} \mu_{12} \mu_{23} \\
& -\mu_{31} \mu_{22} \mu_{13}-\mu_{11} \mu_{32} \mu_{23}-\mu_{33} \mu_{21} \mu_{12} .
\end{aligned}
$$

By Lemma 1.9, the first summand is at least $\frac{8}{27}$, the next two are at least $-\frac{1}{27}$ each, and the latter three are at least $-\frac{2}{27}$ each. Since $8-1-1-2-2-2=0$, in order for $M$ to be singular, each summand must attain its lower bound. This is not possible, since the equality $\frac{2}{3}=1-\lambda_{2}=1-\lambda_{4}$ holds only when $\lambda_{2}=\lambda_{4}=\frac{1}{3}$, in which case $\mu_{13}=\lambda_{2}-\lambda_{4}=0$. Thus the third summand is zero, which is above the required lower bound.

Lemma 2.17. $|B| \leq 6$.

Proof. Subtracting the equalities in Lemma 2.11 for $j=1$ and $j=-1$, we observe that a non-trivial linear combination of $a_{1}-a_{-1}, a_{2}-a_{-2}$, and $a_{3}-a_{-3}$ equals to zero. Hence Lemma 2.16 applies.

\subsection{Sakuma's theorem}

We state Sakuma's theorem.

Theorem 2.18. Let $(V, A,(),, \cdot)$ be a quadruple satisfying (M1) to (M7) in Section 1. Let $a_{0}, a_{1} \in A$ be a pair of Majorana axes, let $\tau_{0}=\tau\left(a_{0}\right), \tau_{1}=\tau\left(a_{1}\right)$ be the corresponding Majorana involutions, let $D$ be the subgroup in $G L(V)$ generated by $\tau_{0}$ and $\tau_{1}$, and let $U$ be the subalgebra in $(V, \cdot)$ generated by $a_{0}$ and $a_{1}$, so that

$$
\left(D, U,\left\{a_{0}, a_{1}\right\},\left.(,)\right|_{U},\left.\cdot\right|_{U}\right)
$$

is a Majorana representation of $D$. Then 
(i) $\operatorname{dim}(U) \leq 8$;

(ii) the isomorphism type of $\left(U,\left.(,)\right|_{U},,\left.\cdot\right|_{U}\right)$ is uniquely determined by the pair $\left(\lambda_{1}, \lambda_{2}\right)$, where $\lambda_{1}=\left(a_{0}, a_{1}\right)$ and $\lambda_{2}=\left(a_{0}, a_{0}^{\tau_{1}}\right)$;

(iii) the existing representations have parameters given in Table 2.

Table 2

\begin{tabular}{|c|ccccccccc|}
\hline & $1 A$ & $2 A$ & $2 B$ & $3 A$ & $3 C$ & $4 A$ & $4 B$ & $5 A$ & $6 A$ \\
\hline$B \mid$ & 1 & 2 & 2 & 3 & 3 & 4 & 4 & 5 & 6 \\
$\lambda_{1}$ & 1 & $\frac{1}{2^{3}}$ & 0 & $\frac{13}{2^{8}}$ & $\frac{1}{2^{6}}$ & $\frac{1}{2^{5}}$ & $\frac{1}{2^{6}}$ & $\frac{3}{2^{7}}$ & $\frac{5}{2^{8}}$ \\
$\lambda_{2}$ & 1 & 1 & 1 & $\frac{13}{2^{8}}$ & $\frac{1}{2^{6}}$ & 0 & $\frac{1}{2^{3}}$ & $\frac{3}{2^{7}}$ & $\frac{13}{2^{8}}$ \\
$\operatorname{dim}(U)$ & 1 & 3 & 2 & 4 & 3 & 5 & 5 & 6 & 8 \\
\hline
\end{tabular}

Proof. The assertion (i) follows from Lemma 2.14 while (ii) is by the proofs of Lemmas 2.10 and 2.14. By Lemma 2.16 we know that $|B| \leq 6$ and the six possible values for $|B|$ will be considered separately. If $|B|$ is odd then all non-zero indexed $\lambda_{j}$ are equal by Lemma 2.9, otherwise $\lambda_{2}$ is known by induction (through considering the subalgebra generated by $a_{0}$ and $a_{2}$ ). As soon as $\lambda_{1}$ and $\lambda_{2}$ are known, the value of $n:=|B|$ can be computed as the smallest positive integer such that $\lambda_{n}=1$. Of course $\operatorname{dim}(U)$ is the rank of the Gram matrix of $X$ computable by Lemma 2.15.

If $|B|=1$ then $a_{0}=a_{1}$ and the algebra is 1 -dimensional, spanned by $a_{0}$ with $a_{0} \cdot a_{0}=a_{0}$ and $\left(a_{0}, a_{0}\right)=1$.

If $|B|=2$ then $a_{0}^{\tau_{1}}=a_{0}, a_{1}^{\tau_{0}}=a_{1}$, so that $\lambda_{2}=1$ and $\lambda_{3}=\lambda_{1}$. Since $\lambda_{3}$ is a polynomial in $\lambda_{1}$ and $\lambda_{2}$ as in Lemma 2.15, the equality $\lambda_{1}=\lambda_{3}$ provides us with a cubic equation on $\lambda_{1}$. This equation has three roots, which are $0, \frac{1}{2^{3}}$ and 1 . Since $a_{0} \neq a_{1}$, the latter root has to be excluded and we obtain the listed pair of values for $\lambda_{1}$.

To deal with higher values of $|B|$, we shall use the following equality implied by Lemma 2.11. For $j$ even,

$$
3 \sigma_{1} \cdot \sigma_{1}+b_{0}\left(\lambda_{1}\right) \sigma_{1}+b_{1} \sigma_{2,0}+b_{2} \sigma_{2,1}=c_{0}\left(\lambda_{1}\right) a_{j}+c_{1}\left(\lambda_{1}\right)\left(a_{j-1}+a_{j+1}\right)+c_{2}\left(a_{j-2}+a_{j+2}\right)
$$


where

$$
\begin{gathered}
b_{0}\left(\lambda_{1}\right)=\left(\frac{5}{2^{2}} \lambda_{1}+\frac{13}{2^{9}}\right), \quad b_{1}=\frac{7}{2^{9}}, \quad b_{2}=-\frac{21}{2^{11}} \\
c_{0}\left(\lambda_{1}\right)=\frac{7}{2} \lambda_{1}^{2}-\frac{7}{2^{7}} \lambda_{1}+\frac{7}{2^{9}} \lambda_{2}-\frac{7}{2^{15}}, \quad c_{1}\left(\lambda_{1}\right)=\frac{49}{2^{8}} \lambda_{1}-\frac{245}{2^{16}}, \quad \text { and } \quad c_{2}=\frac{49}{2^{16}} .
\end{gathered}
$$

If $|B|=3$ then we write (14) for $j=0$ and $j=2$, and take the difference. Using that $a_{4}=a_{1}=a_{-2}, a_{3}=a_{0}$, and $a_{-1}=a_{2}$, we obtain $\left(c_{0}\left(\lambda_{1}\right)-c_{1}\left(\lambda_{1}\right)-c_{2}\right)\left(a_{2}-a_{0}\right)=0$. Since $a_{0} \neq a_{2}$, it follows that $c_{0}\left(\lambda_{1}\right)-c_{1}\left(\lambda_{1}\right)-c_{2}=0$. Since $\lambda_{1}=\lambda_{2}$ by Lemma 2.9, $c_{0}\left(\lambda_{1}\right)-c_{1}\left(\lambda_{1}\right)-c_{2}=0$ is equivalent to the quadratic equation

$$
\lambda_{1}^{2}-\frac{17}{2^{8}} \lambda_{1}+\frac{13}{2^{15}}=0
$$

with the two roots $\frac{13}{2^{8}}$ and $\frac{1}{2^{6}}$.

If $|B|=4$ then $\left|B_{0}\right|=2$ and $\lambda_{2}$ is either 0 or $\frac{1}{2^{3}}$ by induction. Again, we write (14) for $j=0$ and $j=2$, and take the difference. Using that $a_{4}=a_{0}, a_{3}=a_{-1}$, and $a_{-2}=a_{2}$, we obtain $\left(c_{0}\left(\lambda_{1}\right)-2 c_{2}\right)\left(a_{2}-a_{0}\right)=0$ and $c_{0}\left(\lambda_{1}\right)-2 c_{2}=0$. The latter equation is equivalent to

$$
\lambda_{1}^{2}-\frac{1}{2^{6}} \lambda_{1}-c=0,
$$

where $c$ is $\frac{1}{2^{11}}$ or 0 depending on whether $\lambda_{2}$ is 0 or $\frac{1}{2^{3}}$. Since $\lambda_{1} \geq 0$ by Lemma 1.9 and because the pair $\lambda_{1}=0, \lambda_{2}=\frac{1}{2^{3}}$ would give $\lambda_{4}=\frac{173}{8} \neq 1$ in Lemma 2.15, we conclude that $\lambda_{1}$ must be the positive root of (15) and so $\lambda_{1}=\frac{1}{2^{5}}$ or $\frac{1}{2^{6}}$, as claimed.

If $|B|=5$ then writing (14) for $j=0$ and $j=2$, taking the difference, and using $a_{3}=a_{-2}, a_{4}=a_{-1}$ we obtain

$$
\left(c_{0}\left(\lambda_{1}\right)-c_{2}\right)\left(a_{0}-a_{2}\right)+\left(c_{1}\left(\lambda_{1}\right)-c_{2}\right)\left(a_{-1}-a_{-2}\right)=0 .
$$

Taking the inner product of (16) with $a_{0}$ gives $\left(c_{0}\left(\lambda_{1}\right)-c_{2}\right)\left(1-\lambda_{2}\right)+\left(c_{1}\left(\lambda_{1}\right)-c_{2}\right)\left(\lambda_{1}-\right.$ $\left.\lambda_{2}\right)=0$. Here $\lambda_{1}=\lambda_{2}$ by Lemma 2.9 and $1 \neq \lambda_{2}$, so $c_{0}\left(\lambda_{1}\right)=c_{2}$. Substituting back to (16) we get $\left(c_{1}\left(\lambda_{1}\right)-c_{2}\right)\left(a_{-1}-a_{-2}\right)=0$ and then $c_{1}\left(\lambda_{1}\right)=c_{2}$. The latter equation gives $\lambda_{1}=\frac{3}{2^{7}}$.

If $|B|=6$ then writing (14) for $j=0$ and $j=2$, taking the difference, and using $a_{4}=a_{-2}$ we obtain

$$
\left(c_{0}\left(\lambda_{1}\right)-c_{2}\right)\left(a_{0}-a_{2}\right)+c_{1}\left(\lambda_{1}\right)\left(a_{-1}-a_{3}\right)=0 .
$$

Taking the inner product of (17) with $a_{0}$ gives

$$
\left(c_{0}\left(\lambda_{1}\right)-c_{2}\right)\left(1-\lambda_{2}\right)+c_{1}\left(\lambda_{1}\right)\left(\lambda_{1}-\lambda_{3}\right)=0
$$


There are four possibilities for the pair $\left(\lambda_{2}, \lambda_{3}\right)$ by induction, and each of them reduces (18) to a quadratic equation for $\lambda_{1}$. Substituting the solutions $\left(\lambda_{1}, \lambda_{2}\right)$ of these equations into the formula for $\lambda_{3}$ in Lemma 2.15, we see that only one pair gives the correct $\lambda_{3}$ value: $\lambda_{1}=\frac{5}{2^{8}}$ and $\lambda_{2}=\frac{13}{2^{8}}$. (In this case, $\lambda_{3}=\frac{1}{2^{3}}$.)

This completes the proof of Sakuma's theorem.

\subsection{A novelty}

Viewing $\lambda_{1}$ and $\lambda_{2}$ as real parameters, we have a two-parameter family $S\left(\lambda_{1}, \lambda_{2}\right)$ of the algebras with bilinear form on the vector space $W$ spanned by

$$
X=\left\{a_{-2}, a_{-1}, a_{0}, a_{1}, a_{2}, \sigma_{1}, \sigma_{2,0}, \sigma_{2,1}\right\} .
$$

By Theorem 3.7 in [Sak07], both the inner and the algebra products are uniquely determined by $\lambda_{1}$ and $\lambda_{2}$. This statement can be given an explicit form. Indeed, for the inner products we have $\left(a_{j}, a_{j}\right)=\lambda_{|i-j|}$ where $\lambda_{0}=1, \lambda_{1}$ and $\lambda_{2}$ are the given variables, while $\lambda_{3}$ and $\lambda_{4}$ are stated in Lemma 2.15. Lemma 2.15 also gives the inner products between the three $\sigma$ 's. Finally by Lemmas 2.4 and 2.5, and by the associativity between the inner and algebra products we have

$$
\left(a_{i}, \sigma_{1}\right)=\frac{31 \lambda_{1}}{2^{5}}-\frac{1}{2^{5}},\left(a_{2 i+\varepsilon}, \sigma_{2, \varepsilon}\right)=\frac{31 \lambda_{2}}{2^{5}}-\frac{1}{2^{5}},\left(a_{2 i+\varepsilon}, \sigma_{2, \varepsilon+1}\right)=\frac{15 \lambda_{1}}{2^{4}}+\frac{\lambda_{2}}{2^{5}}-\frac{1}{2^{5}} .
$$

In order to express all products of the elements of $X$ as linear combinations of the vectors in $X$, we have computed in [GAP4] the explicit formulas involved in the proofs of Lemmas 2.10, 2.13, and 2.14. For Lemma 2.10, the explicit formulas are described in equations (7)-(13). For the latter two lemmas, the expressions are too lengthy to be given here. The algebras corresponding to the nine cases are specifications of the algebra $S\left(\lambda_{1}, \lambda_{2}\right)$ obtained by assigning to $\lambda_{1}$ and $\lambda_{2}$ the values from Sakuma's Theorem 2.18(iii). When this procedure was implemented during the preparation of this paper, the story has experienced a dramatic twist, which has totally changed our perception of Sakuma's theorem.

The point is, that some (but not all) fusion rules of Lemma 1.5 were exploited in Subsections 2.1-2.4. Imposing the missing fusion rules (by requesting that certain inner products vanish) brings the possibilities for $\left(\lambda_{1}, \lambda_{2}\right)$ down to the nine values in Theorem 2.18(iii) and the arguments in Subsections 2.5 and 2.6 can be eliminated. More specifically, the situation is as follows.

Let

$$
\widehat{\alpha}_{1}=\sigma_{1}+\left(-\frac{3 \lambda_{1}}{2^{2}}+\frac{1}{2^{5}}\right) a_{0}-\frac{7}{2^{6}}\left(a_{1}+a_{-1}\right)
$$




$$
\widehat{\alpha}_{2}=\sigma_{2,0}+\left(-\frac{3 \lambda_{2}}{2^{2}}+\frac{1}{2^{5}}\right) a_{0}-\frac{7}{2^{6}}\left(a_{2}+a_{-2}\right)
$$

be the projections of $a_{1}$ and $a_{2}$ into the 0-eigenspace $V_{0}^{\left(a_{0}\right)}$ of $a_{0}$ divided by -4 (to make the $\sigma_{1}$-coefficient equal to 1 ), and let

$$
\begin{aligned}
& \widehat{\beta}_{1}=\sigma_{1}+\left(-\lambda_{1}+\frac{1}{2^{5}}\right) a_{0}+\frac{1}{2^{6}}\left(a_{1}+a_{-1}\right), \\
& \widehat{\beta}_{2}=\sigma_{2,0}+\left(-\lambda_{2}+\frac{1}{2^{5}}\right) a_{0}+\frac{1}{2^{6}}\left(a_{2}+a_{-2}\right)
\end{aligned}
$$

be the projections of $a_{1}$ and $a_{2}$ into $V_{\frac{1}{2^{2}}}^{\left(a_{0}\right)}$ divided by 4 . Then, because of the fusion rules, the product $\widehat{\beta}_{1} \cdot \widehat{\beta}_{2}$ belongs to $V_{1}^{\left(a_{0}\right)} \oplus V_{0}^{\left(a_{0}\right)}$. Furthermore, by Lemma 1.4 the projection to the 1-eigenspace is simply $\left(\widehat{\beta}_{1} \cdot \widehat{\beta}_{2}, a_{0}\right) a_{0}$. Thus

$$
\widehat{\alpha}_{0}:=\widehat{\beta}_{1} \cdot \widehat{\beta}_{2}-\left(\widehat{\beta}_{1} \cdot \widehat{\beta}_{2}, a_{0}\right) a_{0}
$$

is a 0-eigenvector of $a_{0}$. By the fusion rules, $\widehat{\alpha}_{2} \cdot \widehat{\alpha}_{2}$ is also a 0-eigenvector of $a_{0}$. By Lemma 1.2, eigenvectors with different eigenvalues are perpendicular. Making use of the explicit form of the algebra $S\left(\lambda_{1}, \lambda_{2}\right)$ and of the inner product values, the following was obtained using the [GAP4] package:

$$
\begin{array}{r}
\left(\widehat{\alpha}_{0}, \widehat{\beta}_{1}\right)=-\frac{276480}{49} \lambda_{1}^{5}+\frac{313344}{49} \lambda_{1}^{4}-\frac{9720}{49} \lambda_{1}^{3} \lambda_{2}-\frac{27936}{49} \lambda_{1}^{3}-\frac{495}{784} \lambda_{1}^{2} \lambda_{2}-\frac{135}{196} \lambda_{1} \lambda_{2}^{2} \\
+\frac{2025}{112} \lambda_{1}^{2}-\frac{4653}{12544} \lambda_{1} \lambda_{2}-\frac{531}{50176} \lambda_{2}^{2}-\frac{405}{1792} \lambda_{1}+\frac{3897}{401408} \lambda_{2}+\frac{351}{401408}=0 \\
\left(\widehat{\alpha}_{1} \cdot \widehat{\alpha}_{1}, \widehat{\beta}_{2}\right)=96 \lambda_{1}^{4}-\frac{213}{2} \lambda_{1}^{3}+\frac{15}{8} \lambda_{1}^{2} \lambda_{2}+\frac{135}{16} \lambda_{1}^{2}+\frac{417}{1024} \lambda_{1} \lambda_{2}+\frac{3}{512} \lambda_{2}^{2}-\frac{225}{1024} \lambda_{1} \\
-\frac{501}{65536} \lambda_{2}+\frac{117}{65536}=0
\end{array}
$$

Proposition 2.19. The system of equations (19), (20) in variables $\left(\lambda_{1}, \lambda_{2}\right)$ has precisely nine solutions as in Theorem 2.18(iii).

Proof. The computations described below were carried out in [GAP4]. Taking the resultant of the two equations with respect to the variable $\lambda_{2}$ gives a polynomial of degree 9 in the variable $\lambda_{1}$, with exactly 8 different solutions. Substituting these 
solutions back into the equations, we obtain quadratic polynomials in $\lambda_{2}$, each with two distinct solutions. The result is a set of sixteen pairs $\left(\lambda_{1}, \lambda_{2}\right)$ from (19) and sixteen pairs from (20); exactly nine pairs occur on both lists.

The important conclusion is that, since the arguments in Subsections 2.5 and 2.6 can be eliminated, Lemma 2.17 can be bypassed in the proof of Sakuma's theorem. Meanwhile Lemma 2.17 is the only place in that proof, where the Norton inequality (M2) has been used (through application of Lemma 2.16, based on Lemma 1.9).

\subsection{Norton-Sakuma algebras}

By Sakuma's theorem 2.18 there are at most nine possibilities for the isomorphism type of an algebra with scalar product generated by a pair (of non-necessarily distinct) Majorana axes. Of the other hand it is known that the Monster algebra contains nine different 2-generated subalgebras. Thus the upper and lower bounds meet to produce the explicit version of Sakuma's theorem which is Theorem 2.18 with (iii) upgraded to 'the existing representations with $a_{0} \neq a_{1}$ are given in Table 3.' 
Table 3

\begin{tabular}{|c|c|c|}
\hline Type & Basis & Products and angles \\
\hline $2 \mathrm{~A}$ & $a_{0}, a_{1}, a_{\rho}$ & $\begin{array}{c}a_{0} \cdot a_{1}=\frac{1}{2^{3}}\left(a_{0}+a_{1}-a_{\rho}\right), a_{0} \cdot a_{\rho}=\frac{1}{2^{3}}\left(a_{0}+a_{\rho}-a_{1}\right) \\
\left(a_{0}, a_{1}\right)=\left(a_{0}, a_{\rho}\right)=\left(a_{1}, a_{\rho}\right)=\frac{1}{2^{3}}\end{array}$ \\
\hline $2 \mathrm{~B}$ & $a_{0}, a_{1}$ & $a_{0} \cdot a_{1}=0,\left(a_{0}, a_{1}\right)=0$ \\
\hline $3 \mathrm{~A}$ & $\begin{array}{l}a_{-1}, a_{0}, a_{1} \\
\quad u_{\rho}\end{array}$ & $\begin{array}{c}a_{0} \cdot a_{1}=\frac{1}{2^{5}}\left(2 a_{0}+2 a_{1}+a_{-1}\right)-\frac{3^{3} .5}{2^{11}} u_{\rho} \\
a_{0} \cdot u_{\rho}=\frac{1}{3^{2}}\left(2 a_{0}-a_{1}-a_{-1}\right)+\frac{5}{2^{5}} u_{\rho} \\
\quad u_{\rho} \cdot u_{\rho}=u_{\rho} \\
\left(a_{0}, a_{1}\right)=\frac{13}{2^{8}},\left(a_{0}, u_{\rho}\right)=\frac{1}{2^{2}},\left(u_{\rho}, u_{\rho}\right)=\frac{2^{3}}{5}\end{array}$ \\
\hline $3 \mathrm{C}$ & $a_{-1}, a_{0}, a_{1}$ & $a_{0} \cdot a_{1}=\frac{1}{2^{6}}\left(a_{0}+a_{1}-a_{-1}\right),\left(a_{0}, a_{1}\right)=\frac{1}{2^{6}}$ \\
\hline $4 \mathrm{~A}$ & $\begin{array}{l}a_{-1}, a_{0}, a_{1} \\
\quad a_{2}, v_{\rho}\end{array}$ & $\begin{array}{c}a_{0} \cdot a_{1}=\frac{1}{2^{6}}\left(3 a_{0}+3 a_{1}+a_{2}+a_{-1}-3 v_{\rho}\right) \\
a_{0} \cdot v_{\rho}=\frac{1}{2^{4}}\left(5 a_{0}-2 a_{1}-a_{2}-2 a_{-1}+3 v_{\rho}\right) \\
v_{\rho} \cdot v_{\rho}=v_{\rho}, a_{0} \cdot a_{2}=0 \\
\left(a_{0}, a_{1}\right)=\frac{1}{2^{5}}, \quad\left(a_{0}, a_{2}\right)=0, \quad\left(a_{0}, v_{\rho}\right)=\frac{3}{2^{3}}, \quad\left(v_{\rho}, v_{\rho}\right)=2\end{array}$ \\
\hline $4 \mathrm{~B}$ & $\begin{array}{l}a_{-1}, a_{0}, a_{1} \\
\quad a_{2}, a_{\rho^{2}}\end{array}$ & $\begin{array}{c}a_{0} \cdot a_{1}=\frac{1}{2^{6}}\left(a_{0}+a_{1}-a_{-1}-a_{2}+a_{\rho^{2}}\right) \\
a_{0} \cdot a_{2}=\frac{1}{2^{3}}\left(a_{0}+a_{2}-a_{\rho^{2}}\right) \\
\left(a_{0}, a_{1}\right)=\frac{1}{2^{6}}, \quad\left(a_{0}, a_{2}\right)=\left(a_{0}, a_{\rho}\right)=\frac{1}{2^{3}}\end{array}$ \\
\hline $5 \mathrm{~A}$ & $\begin{array}{c}a_{-2}, a_{-1}, a_{0} \\
a_{1}, a_{2}, w_{\rho}\end{array}$ & $\begin{array}{c}a_{0} \cdot a_{1}=\frac{1}{2^{7}}\left(3 a_{0}+3 a_{1}-a_{2}-a_{-1}-a_{-2}\right)+w_{\rho} \\
a_{0} \cdot a_{2}=\frac{1}{2^{7}}\left(3 a_{0}+3 a_{2}-a_{1}-a_{-1}-a_{-2}\right)-w_{\rho} \\
a_{0} \cdot w_{\rho}=\frac{7}{2^{12}}\left(a_{1}+a_{-1}-a_{2}-a_{-2}\right)+\frac{7}{2^{5}} w_{\rho} \\
w_{\rho} \cdot w_{\rho}=\frac{5^{2} \cdot 7}{2^{19}}\left(a_{-2}+a_{-1}+a_{0}+a_{1}+a_{2}\right) \\
\left(a_{0}, a_{1}\right)=\frac{3}{2^{7}},\left(a_{0}, w_{\rho}\right)=0,\left(w_{\rho}, w_{\rho}\right)=\frac{5^{3} \cdot 7}{2^{19}}\end{array}$ \\
\hline $6 \mathrm{~A}$ & $\begin{array}{l}a_{-2}, a_{-1}, a_{0} \\
\quad a_{1}, a_{2}, a_{3} \\
\quad a_{\rho^{3}}, u_{\rho^{2}}\end{array}$ & $\begin{array}{c}a_{0} \cdot a_{1}=\frac{1}{2^{6}}\left(a_{0}+a_{1}-a_{-2}-a_{-1}-a_{2}-a_{3}+a_{\rho^{3}}\right)+\frac{3^{2} .5}{2^{11}} u_{\rho^{2}} \\
a_{0} \cdot a_{2}=\frac{1}{2^{5}}\left(2 a_{0}+2 a_{2}+a_{-2}\right)-\frac{3^{3} .5}{2^{11}} u_{\rho^{2}} \\
a_{0} \cdot u_{\rho^{2}}=\frac{1}{3^{2}}\left(2 a_{0}-a_{2}-a_{-2}\right)+\frac{5}{2^{5}} u_{\rho^{2}} \\
a_{0} \cdot a_{3}=\frac{1}{2^{3}}\left(a_{0}+a_{3}-a_{\rho^{3}}\right), a_{\rho^{3}} \cdot u_{\rho^{2}}=0,\left(a_{\rho^{3}}, u_{\rho^{2}}\right)=0 \\
\left(a_{0}, a_{1}\right)=\frac{5}{2^{8}},\left(a_{0}, a_{2}\right)=\frac{13}{2^{8}},\left(a_{0}, a_{3}\right)=\frac{1}{2^{3}}\end{array}$ \\
\hline
\end{tabular}


Although Table 3 does not show all pairwise inner and algebra products of the basis vectors, the missing products can be reconstructed by applying symmetries of algebras and their mutual inclusions. The necessary information is given in the following lemma.

Lemma 2.20. Let $S=(U,(),, \cdot)$ be an algebra of type $N X$ in Table 3 , generated by Majorana axes $a_{0}$ and $a_{1}$ (where $N \in\{2,3,4,5,6\}$ and $X \in\{A, B, C\}$ ). Then

(i) $\tau\left(a_{0}\right)$ and $\tau\left(a_{1}\right)$ generate in $G L(V)$ a dihedral group $D$ of order $2 N$ which acts on $U$ with kernel $Z(D)$;

(ii) if $N \geq 3$ then Aut $(S)$ contains a subgroup inducing on $U$ the dihedral group of order $2 N$ on the set $\left\{\ldots, a_{-1}, a_{0}, a_{1}, \ldots\right\}$ of Majorana axes;

(iii) Aut $(2 A)$ contains an $S_{3}$-subgroup acting naturally on $\left\{a_{0}, a_{1}, a_{\rho}\right\}$, while Aut $(2 B)$ contains an element which swaps $a_{0}$ and $a_{1}$;

(iv) $a_{0}$ and $a_{2}$ generate a $2 B$-, $2 A$ - or $3 A$-subalgebra in the algebra of type $4 A, 4 B$ or $6 A$, respectively;

(v) $a_{0}$ and $a_{3}$ generate a $2 A$-subalgebra in the algebra of type $6 \mathrm{~A}$.

Proof. This lemma is implicit in [N96]. Alternatively, the inclusions can be seen from the explicit formulas for multiplying algebra elements that we have computed in the proof of Theorem 2.18. Moreover, the required symmetries are seen from the action of $D=\left\langle\tau_{0}, \tau_{1}\right\rangle$ on the set $B$ of Majorana axes together with the manifestal symmetry between $a_{0}$ and $a_{1}$. The $S_{3}$-symmetry of the $2 A$-type algebra is generated by the automorphisms $\sigma\left(a_{0}\right)$ and $\sigma\left(a_{1}\right)$ as in (M7).

The products in Table 3 are given in the Norton basis inherited from the Monster algebra. An algebra is said to be of type $K$ if it is isomorphic to the subalgebra of the Conway-Griess-Norton Monster algebra generated by a pair of Majorana axes such that the product of the corresponding Majorana involutions is contained in the conjugacy class $K$ of the Monster. In fact, there is no need to know anything about the Monster in order to work with these formulas. One can treat the Norton basis as some basis. The transformation rules towards the original Sakuma basis formed by a subset of $X=\left\{a_{0}, a_{1}, a_{-1}, a_{2}, a_{-2}, \sigma_{1}, \sigma_{2,0}, \sigma_{2,1}\right\}$ can be easily deduced from the products of the Majorana axial vectors. For instance, in the $3 A$-type

$$
\sigma_{1}:=a_{0} \cdot a_{1}-\frac{1}{2^{5}}\left(a_{0}+a_{1}\right)=\frac{1}{2^{5}}\left(a_{0}+a_{1}+a_{-1}\right)-\frac{3^{3} \cdot 5}{2^{11}} u_{\rho}
$$

and hence

$$
u_{\rho}=\frac{2^{6}}{3^{3} \cdot 5}\left(a_{0}+a_{1}+a_{-1}-2^{5} \sigma_{1}\right) .
$$


The Norton basis has to the following important feature. Consider the subalgebra generated by $a_{0}$ and $a_{1}$ in the algebra associated with the Monster group $M$. Let $\tau_{0}$ and $\tau_{1}$ be the $2 A$-involutions associated with $a_{0}$ and $a_{1}$, respectively, and let $\rho=\tau_{0} \tau_{1}$. If the subalgebra generated by $a_{0}$ and $a_{1}$ has type $2 A, 3 A, 4 A$ or $5 A$, then the 1dimensional subspace spanned by the vectors $a_{\rho}, u_{\rho}, v_{\rho}$ or $w_{\rho}$ (in the corresponding Norton basis) is invariant under the normalizer $N_{M}(\langle\rho\rangle)$ isomorphic to $2 \cdot B M, 3 \cdot F_{24}$, $2_{+}^{1+24} \cdot \mathrm{Co}_{3}$ or $\left(D_{10} \times F_{5}\right) .2$, respectively. Furthermore, in the types $2 \mathrm{~A}, 3 \mathrm{~A}$ and $4 \mathrm{~A}$ the vector itself is stable under $N_{M}(\langle\rho\rangle)$, while in the type $5 A$ it is preserved up to negation and satisfies the following:

$$
w_{\rho}=-w_{\rho^{2}}=-w_{\rho^{3}}=w_{\rho^{4}} .
$$

Thus Aut $(5 A)$ contains a Frobenius subgroup of order 20 acting naturally on $\left\{a_{-2}, a_{-1}, a_{0}, a_{1}, a_{2}\right\}$ with a $D_{10}$-subgroup centralizing $w_{\rho}$ and the remaining elements negating this vector.

For each of the four classes the subgroup $\langle\rho\rangle$ is fully normalized in $M$, so that $u_{\rho^{-1}}=u_{\rho}$ and $v_{\rho^{-1}}=v_{\rho}$. The vector $a_{\rho}$ in the $2 A$-type algebra is precisely the Majorana axial vector associated with $\rho$. The vectors $a_{\rho^{2}}, a_{\rho^{3}}$, and $u_{\rho^{2}}$ in the $4 B-$, $6 A-$, and $6 A$-algebras can now be understood in terms of their $2 A-, 2 A$-, and $3 A-$ subalgebras.

It should be emphasized that our scaling of inner and algebra products and our choice of the vectors $a_{\rho}, u_{\rho}, v_{\rho}$ and $w_{\rho}$ differ both from those in [N96]. We have taken $a_{\rho}, u_{\rho}, v_{\rho}$ to be idempotents with the former one having scalar square equal to 1 . The exact numerology is the following: Norton's inner product is 16 times ours, and his $t_{0}, u, v$, and $w$ are $64,90,192$, and 8192 times our $a_{0}, u_{\rho}, v_{\rho}$, and $w_{\rho}$, respectively. Our scaling for the vector $w_{\rho}$ is somewhat arbitrary, since none of its non-zero scalar multiples is an idempotent.

In Table 4 we summarise $a_{0}$-eigenvectors in the Norton-Sakuma algebras (for the eigenvector with eigenvalue 1 we can always take $a_{0}$ itself). 
Table 4

\begin{tabular}{|c|c|c|c|}
\hline Type & 0 & $\frac{1}{2^{2}}$ & $\frac{1}{2^{5}}$ \\
\hline $2 \mathrm{~A}$ & $a_{1}+a_{\rho}-\frac{1}{2^{2}} a_{0}$ & $a_{1}-a_{\rho}$ & \\
\hline $2 \mathrm{~B}$ & $a_{1}$ & & \\
\hline $3 \mathrm{~A}$ & $u_{\rho}-\frac{2 \cdot 5}{3^{3}} a_{0}+\frac{2^{5}}{3^{3}}\left(a_{1}+a_{-1}\right)$ & $u_{\rho}-\frac{2^{3}}{3^{2} \cdot 5} a_{0}-\frac{2^{5}}{3^{2} \cdot 5}\left(a_{1}+a_{-1}\right)$ & $a_{1}-a_{-1}$ \\
\hline $3 \mathrm{C}$ & $a_{1}+a_{-1}-\frac{1}{2^{5}} a_{0}$ & & $a_{1}-a_{-1}$ \\
\hline $4 \mathrm{~A}$ & $v_{\rho}-\frac{1}{2} a_{0}+2\left(a_{1}+a_{-1}\right)+a_{2}, a_{2}$ & $v_{\rho}-\frac{1}{3} a_{0}-\frac{2}{3}\left(a_{1}+a_{-1}\right)-\frac{1}{3} a_{2}$ & $a_{1}-a_{-1}$ \\
\hline $4 \mathrm{~B}$ & $\begin{array}{c}a_{1}+a_{-1}-\frac{1}{2^{5}} a_{0}-\frac{1}{2^{3}}\left(a_{\rho^{2}}-a_{2}\right) \\
a_{2}+a_{\rho^{2}}-\frac{1}{2^{2}} a_{0}\end{array}$ & $a_{2}-a_{\rho^{2}}$ & $a_{1}-a_{-1}$ \\
\hline $5 \mathrm{~A}$ & $\begin{array}{l}w_{\rho}+\frac{3}{2^{9}} a_{0}-\frac{3 \cdot 5}{2^{7}}\left(a_{1}+a_{-1}\right)-\frac{1}{2^{7}}\left(a_{2}+a_{-2}\right) \\
w_{\rho}-\frac{3}{2^{9}} a_{0}+\frac{1}{2^{7}}\left(a_{1}+a_{-1}\right)+\frac{3 \cdot 5}{2^{7}}\left(a_{2}+a_{-2}\right)\end{array}$ & $w_{\rho}+\frac{1}{2^{7}}\left(a_{1}+a_{-1}-a_{2}-a_{-2}\right)$ & $\begin{array}{l}a_{1}-a_{-1} \\
a_{2}-a_{-2}\end{array}$ \\
\hline $6 \mathrm{~A}$ & $\begin{array}{c}u_{\rho^{2}}+\frac{2}{3^{2} \cdot 5} a_{0}-\frac{2^{8}}{3^{2} \cdot 5}\left(a_{1}+a_{-1}\right) \\
-\frac{2^{5}}{3^{2} \cdot 5}\left(a_{2}+a_{-2}+a_{3}-a_{\rho^{3}}\right), \\
a_{3}+a_{\rho^{3}}-\frac{1}{2^{2}} a_{0}, u_{\rho^{2}}-\frac{2 \cdot 5}{3^{3}} a_{0}+\frac{2^{5}}{3^{3}}\left(a_{2}+a_{-2}\right)\end{array}$ & $\begin{array}{l}u_{\rho^{2}}-\frac{2^{3}}{3^{2} .5} a_{0} \\
-\frac{2^{5}}{3^{2} .5}\left(a_{2}+a_{-2}+a_{3}-a_{\rho^{3}}\right), \\
a_{3}-a_{\rho^{3}}\end{array}$ & $\begin{array}{l}a_{1}-a_{-1} \\
a_{2}-a_{-2}\end{array}$ \\
\hline
\end{tabular}

\section{External Majorana representations}

Two-generated subalgebras of type $2 A, 4 B$ and $6 A$ contain further Majorana axes besides the images of the generators under the corresponding dihedral group. We are going to include this feature in the definition.

Let $G$ be a finite group. Let $T$ be a generating set of involutions in $G$ which is a union of some conjugacy classes of $G$. Let $V$ be a real vector space equipped with a positive definite bilinear form ( , ) and a bilinear commutative non-associative algebra product · satisfying (M1) and (M2). Let

$$
\varphi: G \rightarrow G L(V)
$$


be a faithful representation and let

$$
\psi: T \rightarrow V \backslash\{0\}
$$

be a mapping, such that $\psi(t)$ is a Majorana axis for every $t \in T$. Suppose further that

(a) if $\tau(\psi(t))$ is the Majorana involution defined as in (M6) then

$$
\tau(\psi(t))=\varphi(t),
$$

and if $g \in G$ conjugates $t_{1} \in T$ onto $t_{2} \in T$ then $\varphi(g)$ maps $\psi\left(t_{1}\right)$ onto $\psi\left(t_{2}\right)$.

Thus we require that $\varphi(G)$ permutes $\psi(T)$, the same way as the conjugation action of $G$ permutes $T$ (since $\varphi$ is faithful, (a) implies that $\psi$ is injective).

This gives an external version of a Majorana representation (as the closure of $\psi(T)$ with respect to the algebra product). The dimension of this closure is said to be the dimension of the representation.

In the Monster algebra, any three Majorana axes corresponding to a $2 A$-pure elementary abelian subgroup of order four generate a 3 -dimensional subalgebra coinciding with the subalgebra generated by any two of the axes (and having type $2 A$ ). It does not appear easy (if at all possible) to deduce this property from (M1)-(M7). Therefore, we consider this property as an axiom and include it into the definition of an external Majorana representation.

(M8) Let $t_{0}, t_{1}, t_{2} \in T$ and let $a_{i}=\psi\left(t_{i}\right)$ for $0 \leq i \leq 2$. If $a_{0}$ and $a_{1}$ generate a $2 A$ type subalgebra, then $t_{0} t_{1} \in T$ and $\psi\left(t_{0} t_{1}\right)=a_{\rho}$. If $t_{0} t_{1} t_{2}=1$ then the subalgebra generated by $a_{0}$ and $a_{1}$ is of type $2 A$ and $a_{2}=a_{\rho}$.

By Lemma 2.20(iv),(v), the $4 B$ - and $6 A$-type algebras contain $2 A$-subalgebras. Hence axiom (M8) implies the following:

If $a_{0}$ and $a_{1}$ generate a subalgebra of type $2 A, 4 B$, or $6 A$, then $t_{0} t_{1},\left(t_{0} t_{1}\right)^{2}$ or $\left(t_{0} t_{1}\right)^{3}$ belongs to $T$, and $\psi\left(t_{0} t_{1}\right), \psi\left(\left(t_{0} t_{1}\right)^{2}\right)$, or $\psi\left(\left(t_{0} t_{1}\right)^{3}\right)$ coincides with $a_{\rho}, a_{\rho^{2}}$, or $a_{\rho^{3}}$, respectively.

For a Majorana representation it is useful to define its shape by specifying the isomorphism type of the subalgebra generated by $\psi\left(t_{0}\right)$ and $\psi\left(t_{1}\right)$ for every pair $t_{0}, t_{1}$ of involutions in $T$. The shape is subject to various constrains imposed by the $G$-invariance of $T$, as well as by inclusions between subgroups in $G$ generated by various pairs of involutions in $T$ (here (M8) becomes applicable). Effectively, given $G$ and $T$, in order to produce the shape of a possible faithful Majorana representation we have to choose between types $2 A$ and $2 B$ for every $G$-conjugacy class of pairs of commuting involutions in $T$, between types $3 A$ and $3 C$ for every class of pairs giving product of order 3 , and between types $4 A$ and $4 B$ for every class of pairs giving product of order 4 , respecting the inclusions between $T$-generated dihedral subgroups in $G$. For example, if $t_{0}$ and $t_{1}$ are contained in a $T$-generated dihedral group of order 12 and $t_{0} t_{1}$ has order three then $\psi\left(t_{0}\right)$ and $\psi\left(t_{1}\right)$ must generate a 3A-subalgebra. The next lemma is an immediate consequence of (M8). 
Lemma 3.1. For distinct commuting involutions $t_{0}, t_{1} \in T$, the subalgebra generated by $\psi\left(t_{0}\right)$ and $\psi\left(t_{1}\right)$ is of type $2 A$ if $t_{0} t_{1} \in T$, and it is of type $2 B$ otherwise.

We shall call the types $2 B, 2 A, 3 C$, and $4 B$ closed, and the remaining types $3 A, 4 A, 5 A$, and $6 A$ will be called open. This terminology will become clear from the following lemma.

Lemma 3.2. Let

$$
\mathcal{R}=(G, T, V,(,), \cdot, \varphi, \psi)
$$

be a Majorana representation and let $W$ be the linear span of $\psi(T)$. Then

(i) if $t \in T$ and the shape of $\mathcal{R}$ is such that every type involving $t$ is closed, then $W$ is $\psi(t)$-stable;

(ii) if the shape of $\mathcal{R}$ involves closed types only, then $W$ is closed under the algebra multiplication.

Proof. The result is immediate from Table 3.

The last lemma in this section is a direct consequence of Lemma 1.10 in view of Tables 2 and 3 .

Lemma 3.3. Let

$$
\mathcal{R}=(G, T, V,(,), \cdot, \varphi, \psi)
$$

be a Majorana representation, let $t, s \in T$ and suppose that $\psi(t)$ and $\psi(s)$ generate a $2 B$-type algebra (this happens precisely when $[t, s]=1$ and $t s \notin T)$. Then $\psi(t)$ and $\psi(s)$ associate in the sense that

$$
\psi(t) \cdot(v \cdot \psi(s))=(\psi(t) \cdot v) \cdot \psi(s)
$$

for all $v \in V$.

\section{Majorana representations of $S_{4}$}

Let $S_{4}$ be the symmetric group of the set $\Omega=\{i, j, k, l\}$, let $T$ be a generating union of conjugacy classes of involutions in $S_{4}$, so that $T$ is either the set of six transpositions in $S_{4}$, or the total set of all nine involutions in $S_{4}$. Let

$$
\mathcal{S}=\left(S_{4}, T, V,(,), \cdot, \varphi, \psi\right)
$$

be a Majorana representation of $S_{4}$. For $t \in T$ put $a_{t}=\psi(t)$, so that $a_{(i j)}$ is always a Majorana axis while $a_{(i j)(k l)}$ is a Majorana axis if and only if $|T|=9$. Let $F$ denote the isomorphism type of the subalgebra generated by $a_{(i j)}$ and $a_{(k l)}$, and let $E$ denote the isomorphism type of the subalgebra generated by $a_{(i j)}$ and $a_{(i k)}$. We have $F \in\{2 A, 2 B\}$ and $E \in\{3 A, 3 C\}$.

Lemma 4.1. The pair $(F, E)$ determines the whole shape of $\mathcal{S}$. 
Proof. There are two conjugacy classes of pairs of transpositions: the commuting pairs and the pairs with products of order 3. By Lemma 3.1, if $F=2 B$ then $(i j)(k l) \notin T$ and the shape is determined by $F$ and $E$. If $F=2 A$, then $T$ contains all involutions of $S_{4}$ and by Lemma 3.1 the images of any two commuting involutions generate a $2 A$-subalgebra. In this case $a_{(i j)(k l)}$ and $a_{(i k)}$ must generate a $4 B$-subalgebra, since the $4 A$-algebra contains a copy of the $2 B$-algebra.

In what follows the pair $(F, E)$ will be called the shape of $S_{4}$. In view of Lemma 4.1, this should not lead to confusion.

A Majorana representation of $S_{4}$ can be constructed by taking the Majorana representation of the Monster group $M$ and restricting it to a $2 A$-generated $S_{4}$-subgroup. The following proposition has been communicated to us by Simon Norton along with a comment that it can be deduced directly from information in [N98].

Proposition 4.2. Let $\zeta: S_{4} \rightarrow M$ be a monomorphism such that $\zeta((i j))$ is a $2 A$-involution in $M$. Then,

(i) up to $M$-conjugation $\zeta$ is uniquely determined by the $M$-conjugacy classes $F$ and $E$, containing $\zeta((i j)(k l))$ and $\zeta((i j k))$, respectively;

(ii) $\zeta$ exists if and only if $F \in\{2 A, 2 B\}$ and $E \in\{3 A, 3 C\}$. In particular, there are precisely four choices for $\zeta$ (up to $M$-conjugation);

(iii) $C_{M}\left(\zeta\left(S_{4}\right)\right)$ is isomorphic to

$$
2^{11} \cdot M_{23}, \quad S p_{8}(2), \quad{ }^{3} D_{4}(2) \cdot 3, \quad \text { and } 2_{+}^{1+8} \cdot A_{8}
$$

for $(F, E)$ being

$$
(2 B, 3 A), \quad(2 A, 3 A), \quad(2 B, 3 C), \quad \text { and }(2 A, 3 C),
$$

respectively.

There is a standard procedure how to classify the $S_{4}$-subgroups in a given finite group $G$. The procedure is based on the following presentation for $S_{4}$ :

$$
S_{4}=\left\langle x, y, z \mid x^{2}=y^{3}=z^{4}=x y z=1\right\rangle .
$$

The classification is performed separately for every fusion pattern which is the triple $\left(K_{2}, K_{3}, K_{4}\right)$ of conjugacy classes of $G$ containing $x, y$, and $z$, respectively. The number $n\left(K_{2}, K_{3}, K_{4}\right)$ of solutions of the equation

$$
x y z=1
$$

subject to the condition $x \in K_{2}, y \in K_{3}, z \in K_{4}$ can be calculated from the character table of $G$. On the other hand, because of the above presentation for $S_{4}$, we have

$$
\frac{n\left(K_{2}, K_{3}, K_{4}\right)}{|G|}=\sum_{S \in \Xi} \frac{1}{\left|C_{G}(S)\right|},
$$


where $\Xi$ is a transversal of the conjugacy classes of $S_{4}$-subgroups in $G$ with the given fusion pattern. If there exists an $S_{4}$-subgroup $S$ with the considered fusion pattern, and with

$$
\left|C_{G}(S)\right|=\frac{|G|}{n\left(K_{2}, K_{3}, K_{4}\right)},
$$

then all $S_{4}$-subgroups with this fusion pattern are conjugates of $S$.

By taking the monomorphism $\zeta$ as in Proposition 4.2 with $\zeta((i j)(k l)) \in F$ and $\zeta((i j k)) \in$ $E$, followed by the Majorana representation of the Monster, restricted to the image of $\zeta$, we obtain a Majorana representation of $S_{4}$ denoted by $\mathcal{S}_{(F, E)}$. It is clear that $(F, E)$ is the shape of $\mathcal{S}_{(F, E)}$.

The central result of the present paper is the following.

Theorem 4.3. Every Majorana representation of $S_{4}$ is isomorphic to one of the representations

$$
\mathcal{S}_{(2 B, 3 A)}, \quad \mathcal{S}_{(2 A, 3 A)}, \quad \mathcal{S}_{(2 B, 3 C)}, \quad \text { and } \mathcal{S}_{(2 A, 3 C)} .
$$

Because of Lemma 4.1 and Proposition 4.2, in order to prove Theorem 4.3 it is sufficient to justify the following.

Proposition 4.4. The isomorphism type of a Majorana representation of $S_{4}$ is uniquely determined by its shape.

By proving Proposition 4.4 instead of Theorem 4.3, we save verification that the representations indeed satisfy all the Majorana conditions. On the other hand, our explicit construction of the representations $\mathcal{S}_{(F, E)}$ contributes to the knowledge of the Monster algebra structure.

Proposition 4.5. The dimension of the algebra $\mathcal{S}_{(F, E)}$ and the scalar square of its identity element are as given in Table 5.

Table 5

\begin{tabular}{|c|cccc|}
\hline$(F, E)$ & $(2 B, 3 A)$ & $(2 A, 3 A)$ & $(2 B, 3 C)$ & $(2 A, 3 C)$ \\
\hline dimension & 13 & 13 & 6 & 9 \\
scalar square of the identity & $\frac{2^{2} \cdot 47}{5^{2}}$ & $\frac{2^{2} \cdot 3^{2}}{5}$ & $\frac{2^{5} \cdot 3}{17}$ & $\frac{2^{5} \cdot 137}{3 \cdot 5 \cdot 7^{2}}$ \\
\hline
\end{tabular}

Each of the four feasible shapes will be handled in a separate subsection. 


\subsection{Shape $(2 B, 3 A)$}

We start calculating in the Sakuma basis and will switch to the Norton basis in Section 5 . The products of the six Majorana generators (indexed by the $S_{4}$-transpositions) are as follows:

$$
\begin{array}{r}
a_{(i j)} \cdot a_{(i j)}=a_{(i j)} ; \quad a_{(i j)} \cdot a_{(k l)}=0 ; \quad a_{(i j)} \cdot a_{(i k)}=\sigma_{l}+\frac{1}{2^{5}}\left(a_{(i j)}+a_{(i k)}\right) ; \\
\sigma_{l} \cdot \sigma_{l}=-\frac{3^{2} \cdot 7}{2^{11}} \sigma_{l}+\frac{3 \cdot 7^{2}}{2^{16}}\left(a_{(i j)}+a_{(i k)}+a_{(j k)}\right) .
\end{array}
$$

The third of the above equalities can be treated as a definition of the vector $\sigma_{l}$ which is invariant under the $S_{3}$-subgroup stabilizing $l$. The fourth equation is the specialization of (13) for 3A-algebras, with $\sigma_{l}=\sigma_{1}=\sigma_{2,0}=\sigma_{2,1}, a_{(i j)}=a_{0}, a_{(i k)}=a_{1}=a_{-2}, a_{(j k)}=a_{2}=$ $a_{-1}$, and $\lambda_{1}=\lambda_{2}=\frac{13}{2^{8}}$.

It turns out that the linear span of the six $a$ 's and four $\sigma$ 's is not closed under the algebra product: three further dimensions are required.

Lemma 4.6. The vector

$$
\delta_{(i j)(k l)}:=\sigma_{i} \cdot a_{(i j)}-\frac{1}{2^{5}} \sigma_{i}+\frac{1}{2^{10}} a_{(i j)}
$$

depends only on the even involution from $S_{4}$ shown in its index.

Proof. Since $a_{(i j)}$ and $a_{(k l)}$ generate a subalgebra of type $2 B$, by Lemma 3.3 they associate with every element of $V$. In particular

$$
\left(a_{(i j)} \cdot a_{(i k)}\right) \cdot a_{(k l)}=a_{(i j)} \cdot\left(a_{(i k)} \cdot a_{(k l)}\right) .
$$

Making use of the expressions given in (21) and their $S_{4}$-conjugates, we can express both sides of the last equality as linear combinations of $a$ 's, $\sigma$ 's and their products to obtain the equality

$$
\sigma_{l} \cdot a_{(k l)}+\frac{1}{2^{5}} \sigma_{j}+\frac{1}{2^{10}} a_{(k l)}=\sigma_{j} \cdot a_{(i j)}+\frac{1}{2^{5}} \sigma_{l}+\frac{1}{2^{10}} a_{(i j)},
$$

which can be rearranged to

$$
\sigma_{l} \cdot a_{(k l)}-\frac{1}{2^{5}} \sigma_{l}+\frac{1}{2^{10}} a_{(k l)}=\sigma_{j} \cdot a_{(i j)}-\frac{1}{2^{5}} \sigma_{j}+\frac{1}{2^{10}} a_{(i j)} .
$$

Having done this, we observe that every summand on the left hand side is stable under the transposition $\varphi((i j))$, while applying this transposition to the right hand side gives

$$
\sigma_{i} \cdot a_{(i j)}-\frac{1}{2^{5}} \sigma_{i}+\frac{1}{2^{10}} a_{(i j)} .
$$

The involution $\varphi((i k)(j l))$ permutes the left and the right hand sides of $(24)$. Thus $\delta_{(i j)(k l)}$ is invariant under the dihedral subgroup $D$ of order 8 in $\varphi\left(S_{4}\right)$ generated by $\varphi((i j))$ and $\varphi((i k)(j l))$. Since $D=C_{\varphi\left(S_{4}\right)}(\varphi((i j)(k l)))$, the result follows. 
Let $W$ denote the linear span of the six $a$ 's and four $\sigma$ 's and let $U$ be the linear span of $W$ together with the three $\delta$ 's. It is clear that both $W$ and $U$ are $\varphi\left(S_{4}\right)$-invariant submodules. Our first goal is to show that $U$ is $a_{t}$-stable for every $t \in T$ and the ultimate aim is to show that $U$ is closed for the algebra product.

Lemma 4.7. For all $w \in W$ and $t \in T$ we have $a_{t} \cdot w \in U$.

Proof. Because of the product rules given in (21) and by (23), it is enough to show that $a_{(i j)} \cdot \sigma_{k} \in U$. Since $a_{(i j)}$ and $\sigma_{k}$ are contained in a $3 A$-subalgebra, equation (3) in Lemma 2.4, applied with $\lambda_{1}=\frac{13}{2^{8}}, a_{j}=a_{(i j)}$, and $\sigma_{1}=\sigma_{k}$, gives

$$
a_{(i j)} \cdot \sigma_{k}=\frac{7}{2^{5}}\left(\sigma_{k}+\frac{1}{2^{4}} a_{(i j)}+\frac{1}{2^{6}}\left(a_{(i l)}+a_{(j l)}\right)\right) .
$$

The next lemma also contributes to the proof of the $a_{t}$-stability of $U$.

Lemma 4.8. The following equality holds:

$$
a_{(i j)} \cdot \delta_{(i j)(k l)}=\frac{7}{2^{5}}\left[\delta_{(i j)(k l)}+\frac{1}{2^{6}}\left(\sigma_{i}+\sigma_{j}\right)-\frac{1}{2^{9}} a_{(i j)}\right] .
$$

Proof. By Lemma 4.6 we have

$$
a_{(i j)} \cdot \delta_{(i j)(k l)}=a_{(i j)} \cdot\left(\sigma_{k} \cdot a_{(k l)}\right)-\frac{1}{2^{5}} a_{(i j)} \cdot \sigma_{k}+\frac{1}{2^{10}} a_{(i j)} \cdot a_{(k l)} \cdot
$$

Since $a_{(i j)}$ and $a_{(k l)}$ annihilate each other and associate, the last summand is zero, while the first one is equal to

$$
\left(a_{(i j)} \cdot \sigma_{k}\right) \cdot a_{(k l)} \cdot
$$

Expanding $a_{(i j)} \cdot \sigma_{k}$ by (25) and then applying (21) and (23) and simplifying, we obtain the required expression.

For further analysis we require the knowledge of some eigenvectors of the adjoint action of $a_{(i j)}$ on $U$.

Lemma 4.9. The following table shows some 0 - and $\frac{1}{2^{2}}$-eigenvectors (first and the second columns, respectively) of $a_{(i j)}$ acting on $U$, where $\mu=\left(\beta_{k} \cdot \beta_{l}, a_{(i j)}\right)=-\frac{3^{2} \cdot 13}{2^{20}}$. 
Table 6

\begin{tabular}{|c|c|}
\hline 0 & $\frac{1}{2^{2}}$ \\
\hline$\alpha_{k}=\sigma_{k}-\frac{7}{2^{10}} a_{(i j)}-\frac{7}{2^{6}}\left(a_{(i l)}+a_{(j l)}\right)$ & $\beta_{k}=\sigma_{k}-\frac{5}{2^{8}} a_{(i j)}+\frac{1}{2^{6}}\left(a_{(i l)}+a_{(j l)}\right)$ \\
$\alpha_{l}=\sigma_{l}-\frac{7}{2^{10}} a_{(i j)}-\frac{7}{2^{6}}\left(a_{(i k)}+a_{(j k)}\right)$ & $\beta_{l}=\sigma_{l}-\frac{5}{2^{8}} a_{(i j)}+\frac{1}{2^{6}}\left(a_{(i k)}+a_{(j k)}\right)$ \\
$a_{(k l)}$ & \\
$\alpha_{k} \cdot \alpha_{l}=\sigma_{k} \cdot \sigma_{l}-\frac{7}{2^{5}}\left(\delta_{(i k)(j l)}+\delta_{(i l)(j k)}\right) \bmod W$ & $\alpha_{k} \cdot \beta_{l}=\sigma_{k} \cdot \sigma_{l}-\frac{3}{2^{5}}\left(\delta_{(i k)(j l)}+\delta_{(i l)(j k)}\right) \bmod W$ \\
$\beta_{k} \cdot \beta_{l}-\mu a_{(i j)}=\sigma_{k} \cdot \sigma_{l}+\frac{1}{2^{5}}\left(\delta_{(i k)(j l)}+\delta_{(i l)(j k)}\right) \bmod W$ & \\
$\alpha_{(i j)}=\delta_{(i j)(k l)}-\frac{7}{2^{6}}\left(\sigma_{i}+\sigma_{j}\right)+\frac{7}{2^{15}} a_{(i j)}$ & $\beta_{(i j)}=\delta_{(i j)(k l)}+\frac{1}{2^{6}}\left(\sigma_{i}+\sigma_{j}\right)+\frac{5}{2^{13}} a_{(i j)}$ \\
\hline
\end{tabular}

Proof. The eigenvectors $\alpha_{k}$ and $\beta_{k}$ are contained in the $3 A$-subalgebra generated by $a_{(i j)}$ and $a_{(i l)}$, and they are specializations of (1) and (2) in Lemma 2.4 (divided by -4 and 4 , respectively). Similarly $\alpha_{l}$ and $\beta_{l}$ are in the $3 A$-algebra generated by $a_{(i j)}$ and $a_{(i k)}$. Since $a_{(i j)}$ and $a_{(k l)}$ generate a $2 B$-subalgebra, $a_{(k l)}$ is a 0-eigenvector of $a_{(i j)}$. Multiplying $\alpha_{l}$ by $a_{(k l)}$ and subtracting

$$
\frac{1}{2^{5}} \alpha_{l}-\frac{1}{2^{7}} a_{(k l)}
$$

(which is a 0 -eigenvector of $a_{(i j)}$ ) we obtain $\alpha_{(i j)}$ as in Table 6. In a similar way, making use of $\beta_{l}$ instead of $\alpha_{l}$, we obtain $\beta_{(i j)}$.

Lemma 4.10. The following assertions hold:

(i) $a_{(i j)} \cdot\left(\delta_{(i k)(j l)}+\delta_{(i l)(j k)}\right) \in U$;

(ii) $U$ is $a_{(i j)}$-stable;

(iii) $\sigma_{k} \cdot \sigma_{l} \in U$.

Proof. We prove (i) and (ii) by applying a modification of the resurrection principle Lemma 1.7. By Table 6 we have

$$
\begin{aligned}
& \alpha_{k} \cdot \alpha_{l}=\sigma_{k} \cdot \sigma_{l}-\frac{7}{2^{5}}\left(\delta_{(i k)(j l)}+\delta_{(i l)(j k)}\right) \bmod W, \\
& \beta_{k} \cdot \beta_{l}=\sigma_{k} \cdot \sigma_{l}+\frac{1}{2^{5}}\left(\delta_{(i k)(j l)}+\delta_{(i l)(j k)}\right) \bmod W, \\
& \alpha_{k} \cdot \beta_{l}=\sigma_{k} \cdot \sigma_{l}+\frac{1}{2^{5}}\left(\delta_{(i k)(j l)}+\delta_{(i l)(j k)}\right) \bmod W .
\end{aligned}
$$

This shows that

$$
\alpha_{k} \cdot \alpha_{l}-\beta_{k} \cdot \beta_{l}+\left(\beta_{k} \cdot \beta_{l}, a_{(i j)}\right) a_{(i j)}=-\frac{1}{2^{2}}\left(\delta_{(i k)(j l)}+\delta_{(i l)(j k)}\right) \bmod W
$$


is a 0 -eigenvector of $a_{(i j)}$, and so Lemma 4.7 implies (i).

By Lemmas 4.7, 4.8, and the just proven part (i) of this lemma, in order to establish (ii) it is sufficient to show that $U$ contains $a_{(i j)} \cdot\left(\delta_{(i k)(j l)}-\delta_{(i l)(j k)}\right)$. But this is indeed the case, because (M6) implies that $\delta_{(i k)(j l)}-\delta_{(i l)(j k)}$ is a $\frac{1}{2^{5}}$-eigenvector of $a_{(i j)}$, since $\varphi((i j))$ permutes $\delta_{(i k)(j l)}$ and $\delta_{(i l)(j k)}$.

Finally, by (i) and the above expressions we know that both $\alpha_{k} \cdot \alpha_{l}$ and $\alpha_{k} \cdot \beta_{l}$ are equal to $\sigma_{k} \cdot \sigma_{l}$ modulo $U$. This fact and (ii) enable us to apply Lemma 1.8 to establish (iii).

Lemma 4.11. The following assertions hold:

(i) $\sigma_{k} \cdot \delta_{(i j)(k l)} \in U$;

(ii) $\delta_{(i j)(k l)} \cdot \delta_{(i j)(k l)} \in U$;

(iii) $\delta_{(i k)(j l)} \cdot \delta_{(i l)(j k)} \in U$;

(iv) the product $\cdot$ is closed on $U$.

Proof. At each stage we implicitly make use of Lemma 4.10(ii),(iii), as well as of all previous assertions of the present lemma. We apply Lemma 1.8 to different pairs of eigenvalues. For (i) we put $s=\sigma_{k} \cdot \delta_{(i j)(k l)}, \alpha_{s}=\alpha_{k} \cdot \alpha_{(i j)}, \beta_{s}=\alpha_{k} \cdot \beta_{(i j)}$. For (ii) we put $s=\delta_{(i j)(k l)} \cdot \delta_{(i j)(k l)}$, $\alpha_{s}=\alpha_{(i j)} \cdot \alpha_{(i j)}, \beta_{s}=\alpha_{(i j)} \cdot \beta_{(i j)}$. Finally, for (iii) we put

$$
s=\delta_{(i k)(j l)} \cdot \delta_{(i l)(j k)}, \quad \alpha_{s}=\frac{2^{9}}{7^{2}}\left(\alpha_{k} \cdot \alpha_{l}\right) \cdot\left(\alpha_{k} \cdot \alpha_{l}\right), \quad \beta_{s}=\frac{2^{9}}{3.7}\left(\alpha_{k} \cdot \alpha_{l}\right) \cdot\left(\alpha_{k} \cdot \beta_{l}\right) .
$$

Now (iv) holds because of (22), Lemma 4.10(ii),(iii), parts (i)-(iii) of this lemma, and the $S_{4}$-invariance of $U$.

The explicit versions of Lemmas 4.10(i),(iii) and 4.11(i),(ii),(iii)), computed in [GAP4], are as follows:

$$
\begin{gathered}
a_{(i j)} \cdot \delta_{(i k)(j l)}=\frac{1}{2^{16}}\left(a_{(i k)}+a_{(i l)}+a_{(j k)}+a_{(j l)}\right)+\frac{1}{2^{12}}\left(3 \sigma_{i}+3 \sigma_{j}+4 \sigma_{k}+4 \sigma_{l}\right) \\
+\frac{1}{2^{6}}\left(3 \delta_{(i j)(k l)}+\delta_{(i k)(j l)}-\delta_{(i l)(j k)}\right) ; \\
\sigma_{i} \cdot \sigma_{j}=\frac{5}{2^{16}} a_{(k l)}+\frac{1}{2^{16}}\left(a_{(i k)}+a_{(i l)}+a_{(j k)}+a_{(j l)}\right) \\
+\frac{1}{2^{12}}\left(14 \sigma_{i}+14 \sigma_{j}+3 \sigma_{k}+3 \sigma_{l}\right)-\frac{1}{2^{5}}\left(2 \delta_{(i j)(k l)}-3 \delta_{(i k)(j l)}-3 \delta_{(i l)(j k)}\right) ; \\
\sigma_{i} \cdot \delta_{(i j)(k l)}=\frac{1}{2^{23}}\left(2 \cdot 61 a_{(i j)}+2^{3} \cdot 13 a_{(k l)}+5^{2} a_{(i k)}+5^{2} a_{(i l)}+2 \cdot 3 \cdot 17 a_{(j k)}+2 \cdot 3 \cdot 17 a_{(j l)}\right) \\
+\frac{1}{2^{17}}\left(-2^{2} \cdot 17 \sigma_{i}-2 \cdot 3^{2} \sigma_{j}+59 \sigma_{k}+59 \sigma_{l}\right)-\frac{1}{2^{12}}\left(2^{3} \cdot 3 \delta_{(i j)(k l)}-19 \delta_{(i k)(j l)}-19 \delta_{(i l)(j k)}\right) ;
\end{gathered}
$$




$$
\begin{gathered}
\delta_{(i j)(k l)} \cdot \delta_{(i j)(k l)}=\frac{43}{2^{22}}\left(a_{(i j)}+a_{(k l)}\right)+\frac{3^{2} \cdot 41}{2^{27}}\left(a_{(i k)}+a_{(i l)}+a_{(j k)}+a_{(j l)}\right) \\
-\frac{643}{2^{23}}\left(\sigma_{i}+\sigma_{j}+\sigma_{k}+\sigma_{l}\right)+\frac{1}{2^{15}}\left(11 \delta_{(i j)(k l)}+3 \cdot 13 \delta_{(i k)(j l)}+3 \cdot 13 \delta_{(i l)(j k)}\right) \\
\delta_{(i k)(j l)} \cdot \delta_{(i l)(j k)}=\frac{3 \cdot 7 \cdot 13}{2^{27}}\left(a_{(i k)}+a_{(i l)}+a_{(j k)}+a_{(j l)}\right)-\frac{73}{2^{27}}\left(a_{(i j)}+a_{(k l)}\right) \\
+\frac{3 \cdot 13}{2^{24}}\left(\sigma_{i}+\sigma_{j}+\sigma_{k}+\sigma_{l}\right)+\frac{5 \cdot 11}{2^{16}}\left(\delta_{(i k)(j l)}+\delta_{(i l)(j k)}\right)-\frac{3^{2}}{2^{16}} \delta_{(i j)(k l)} .
\end{gathered}
$$

The above expressions, together with the product rules given in (21), (22), (23), (25), and in Lemma 4.8, completely describe the products in the algebra $\mathcal{S}_{(2 B, 3 A)}$. The Gram matrix with respect to the scalar product (, ) in the Sakuma basis was mostly computed by hand, although also checked in [GAP4]. In Table 7, one row from each of the three $S_{4}$-orbits on the basis vectors is shown and $\delta_{(i j)(k l)}$ is abbreviated as $\delta_{j}$.

Table 7

\begin{tabular}{|c|ccccccccccccc|}
\hline$()$, & $a_{(i j)}$ & $a_{(k l)}$ & $a_{(i k)}$ & $a_{(i l)}$ & $a_{(j k)}$ & $a_{(j l)}$ & $\sigma_{i}$ & $\sigma_{j}$ & $\sigma_{k}$ & $\sigma_{l}$ & $\delta_{j}$ & $\delta_{k}$ & $\delta_{l}$ \\
\hline$a_{(i j)}$ & 1 & 0 & $\frac{13}{2^{8}}$ & $\frac{13}{2^{8}}$ & $\frac{13}{2^{8}}$ & $\frac{13}{2^{8}}$ & $-\frac{13}{2^{13}}$ & $-\frac{13}{2^{13}}$ & $\frac{3 \cdot 7^{2}}{2^{13}}$ & $\frac{3 \cdot 7^{2}}{2^{13}}$ & $-\frac{3 \cdot 7^{2}}{2^{18}}$ & $\frac{5}{2^{19}}$ & $\frac{5}{2^{19}}$ \\
$\sigma_{i}$ & $-\frac{13}{2^{13}}$ & $\frac{3 \cdot 7^{2}}{2^{13}}$ & $-\frac{13}{2^{13}}$ & $-\frac{13}{2^{13}}$ & $\frac{3 \cdot 7^{2}}{2^{13}}$ & $\frac{3 \cdot 7^{2}}{2^{13}}$ & $\frac{3^{2} \cdot 7 \cdot 59}{2^{19}}$ & $\frac{5}{2^{19}}$ & $\frac{5}{2^{19}}$ & $\frac{5}{2^{19}}$ & $\frac{449}{2^{23}}$ & $\frac{449}{2^{23}}$ & $\frac{449}{2^{23}}$ \\
$\delta_{j}$ & $-\frac{3 \cdot 7^{2}}{2^{18}}$ & $-\frac{3 \cdot 7^{2}}{2^{18}}$ & $\frac{5}{2^{19}}$ & $\frac{5}{2^{19}}$ & $\frac{5}{2^{19}}$ & $\frac{5}{2^{19}}$ & $\frac{449}{2^{23}}$ & $\frac{449}{2^{23}}$ & $\frac{449}{2^{23}}$ & $\frac{449}{2^{23}}$ & $\frac{5 \cdot 3697}{2^{29}}$ & $\frac{3^{5} \cdot 29}{2^{30}}$ & $\frac{3^{5} \cdot 29}{2^{30}}$ \\
\hline
\end{tabular}

The Gram matrix is non-singular, hence 13 is the dimension of the representation. The determinant of the Gram matrix, as calculated (on the very early stages of this project) by Alexander Osipov from the Institute of Information Systems in Novosibirsk, is

$$
\frac{3^{16} \cdot 5^{2} \cdot 11^{2} \cdot 23^{9}}{2^{152}}
$$

The conceptional meaning of this number is not yet clear for us.

\subsection{Shape $(2 A, 3 A)$}

Here we follow the Norton basis from the very beginning. The products of vectors in $\psi(T)$ are described by the following rules:

$$
\begin{gathered}
a_{(i j)} \cdot a_{(i j)}=a_{(i j)} ; \quad a_{(i j)(k l)} \cdot a_{(i j)(k l)}=a_{(i j)(k l)} \\
a_{(i j)} \cdot a_{(k l)}=\frac{1}{2^{3}}\left(a_{(i j)}+a_{(k l)}-a_{(i j)(k l)}\right)
\end{gathered}
$$




$$
\begin{gathered}
a_{(i j)(k l)} \cdot a_{(i k)(j l)}=\frac{1}{2^{3}}\left(a_{(i j)(k l)}+a_{(i k)(j l)}-a_{(i l)(j k)}\right) \\
a_{(i j)} \cdot a_{(i k)(j l)}=\frac{1}{2^{6}}\left(a_{(i j)}+a_{(i k)(j l)}-a_{(k l)}-a_{(i l)(j k)}+a_{(i j)(k l)}\right) ; \\
a_{(i j)} \cdot a_{(i j)(k l)}=\frac{1}{2^{3}}\left(a_{(i j)}+a_{(i j)(k l)}-a_{(k l)}\right) ; \\
a_{(i j)} \cdot a_{(i k)}=\frac{1}{2^{5}}\left(2 a_{(i j)}+2 a_{(i k)}+a_{(j k)}\right)-\frac{3^{3} .5}{2^{11}} u_{l} ; \\
u_{l} \cdot u_{l}=u_{l} ; \quad a_{(i j)} \cdot u_{l}=\frac{1}{3^{2}}\left(2 a_{(i j)}-a_{(i k)}-a_{(j k)}\right)+\frac{5}{2^{5}} u_{l} .
\end{gathered}
$$

Equations (26)-(30) follow from lines $2 \mathrm{~A}$ and $4 \mathrm{~B}$ of Table 3 (see also the proof of Lemma 4.1). Equation (31) can be considered as the definition of $u_{l}$. The latter vector is invariant under the $\varphi$-image of the symmetric group $S_{3}$ on the letters $i, j, k$. Finally, (32) follows from line $3 \mathrm{~A}$ of Table 3.

Let $W$ denote the linear span of $\psi(T)$ and let $U$ denote the linear span of $W$ together with $u_{i}, u_{j}, u_{k}$, and $u_{l}$. Our goal is to show that $U$ is closed under the algebra multiplication, which will be achieved in a sequence of lemmas.

The first lemma is an immediate consequence of (26)-(32).

Lemma 4.12. The following assertions hold:

(i) $a_{t} \cdot w \in U$ for all $t \in T$ and all $w \in W$;

(ii) $a_{(i j)} \cdot u_{l} \in U$;

(iii) $a_{(i j)(k l)} \cdot a_{t} \in W$ for all $t \in T$.

Lemma 4.13. Table 8 shows some 0 - and $\frac{1}{2^{2}}$-eigenvectors (first and the second columns, respectively) of $a_{(i j)}$.

\section{Table 8}

\begin{tabular}{|c|c|}
\hline 0 & $\frac{1}{2^{2}}$ \\
\hline$\alpha_{(i k)(j l)}=a_{(i k)(j l)}+a_{(i l)(j k)}-\frac{1}{2^{2}} a_{(i j)(k l)}$ & \\
$\alpha_{(k l)}=a_{(k l)}+a_{(i j)(k l)}-\frac{1}{2^{2}} a_{(i j)}$ & $\beta_{(k l)}=a_{(k l)}-a_{(i j)(k l)}$ \\
$\alpha_{l}=u_{l}-\frac{2.5}{3^{3}} a_{(i j)}+\frac{2^{5}}{3^{3}}\left(a_{(i k)}+a_{(j k)}\right)$ & $\beta_{l}=u_{l}-\frac{2^{3}}{3^{2} .5} a_{(i j)}-\frac{2^{5}}{3^{2} .5}\left(a_{(i k)}+a_{(j k)}\right)$ \\
\hline
\end{tabular}


Proof. The result follows from Table 4 in view of the shape of the considered representation.

Lemma 4.14. The following assertions hold:

(i) $\alpha_{(i k)(j l)} \cdot u_{l} \in U$;

(ii) $a_{(i j)(k l)} \cdot u_{l} \in U$;

(iii) $\beta_{(k l)} \cdot u_{l} \in U$;

(iv) $a_{(k l)} \cdot u_{l} \in U$.

Proof. To prove (i), we apply Lemma 1.8 for $s=\alpha_{(i k)(j l)} \cdot u_{l}, \alpha_{s}=\alpha_{(i k)(j l)} \cdot \alpha_{l}, \beta_{s}=$ $\alpha_{(i k)(j l)} \cdot \beta_{l}$, making use of Lemmas 4.12 and 4.13 .

Since $u_{l}$ is stable under the permutation $\varphi((i j k))$, while $\alpha_{(i k)(j l)}$ is not, (i) implies that $U$ contains each of the following three vectors:

$$
\alpha_{(i k)(j l)} \cdot u_{l}, \quad \alpha_{(j k)(i l)} \cdot u_{l}, \quad \alpha_{(i j)(k l)} \cdot u_{l} .
$$

Considering a suitable linear combination of the $\alpha$ 's, we obtain (ii).

To prove (iii) we apply a modification of the resurrection principle. By the just proven part (ii) and Lemma 4.12, the product

$$
\alpha_{l} \cdot\left(\alpha_{(k l)}-\beta_{(k l)}\right)=\alpha_{l} \cdot \alpha_{(k l)}-\alpha_{l} \cdot \beta_{(k l)}
$$

belongs to the linear span of $W$ and $u_{l}$. Since this span is $a_{(i j)}$-stable under the adjoint action of $a_{(i j)}$ (see Lemma 4.12(ii)), and since the above product is a difference of a 0-eigenvector and a $\frac{1}{2^{2}}$-eigenvector of $a_{(i j)}$, (iii) follows from the equality

$$
a_{(i j)} \cdot\left(\alpha_{l} \cdot\left(\alpha_{(k l)}-\beta_{(k l)}\right)\right)=-\frac{1}{2^{2}} \alpha_{l} \cdot \beta_{(k l)} .
$$

Finally, (iv) is an immediate consequence of (ii) and (iii).

By Lemma 4.12(i),(ii) and Lemma 4.14(iv) we have the following.

Lemma 4.15. The subspace $U$ is $a_{t}$-stable for every $t \in T$.

Proposition 4.16. The product $\cdot$ is closed on $U$.

Proof. By Lemma 4.15 and since $u_{l}$ is an idempotent, in order to prove the assertion it is sufficient to show that $U$ contains $u_{l} \cdot u_{k}$. The latter follows from Lemma 1.8 applied to $s=u_{l} \cdot u_{k}, \alpha_{s}=\alpha_{l} \cdot \alpha_{k}$, and $\beta_{s}=\alpha_{l} \cdot \beta_{k}$.

The product formulas, as calculated in [GAP4], are the following:

$$
a_{(i j)} \cdot u_{i}=\frac{1}{3^{2} \cdot 5}\left(a_{(i j)}+a_{(k l)}-a_{(i j)(k l)}\right)-\frac{1}{2 \cdot 3^{2} \cdot 5}\left(a_{(i k)}+a_{(i l)}+a_{(j k)}+a_{(j l)}\right)+\frac{1}{2^{6}}\left(u_{i}-u_{j}+u_{k}+u_{l}\right) ;
$$




$$
\begin{gathered}
a_{(i j)(k l)} \cdot u_{i}=\frac{1}{3^{2}} a_{(i j)(k l)}+\frac{1}{2^{6}}\left(5 u_{i}+3 u_{j}-4 u_{k}-4 u_{l}\right) ; \\
u_{i} \cdot u_{j}=\frac{1}{5}\left(u_{i}+u_{j}\right)-\frac{1}{2 \cdot 3^{2}}\left(u_{k}+u_{l}\right)+\frac{2^{6}}{3^{4} \cdot 5^{2}}\left(2 a_{(i j)(k l)}-3 a_{(i k)(j l)}-3 a_{(i l)(j k)}\right) .
\end{gathered}
$$

The identity element is

$$
\iota=\frac{2^{3}}{3 \cdot 5} \sum_{t \in T} a_{t}+\frac{3}{2^{3}} \sum_{x \in \Omega} u_{x}, \text { and }(\iota, \iota)=\frac{2^{2} \cdot 3^{2}}{5} .
$$

The reduced inner product matrix is the following, where $a_{(i j)(k l)}$ is abbreviated as $a_{j}$.

Table 9

\begin{tabular}{|c|ccccccccccccc|}
\hline$()$, & $a_{(i j)}$ & $a_{(k l)}$ & $a_{(i k)}$ & $a_{(i l)}$ & $a_{(j k)}$ & $a_{(j l)}$ & $u_{i}$ & $u_{j}$ & $u_{k}$ & $u_{l}$ & $a_{j}$ & $a_{k}$ & $a_{l}$ \\
\hline$a_{(i j)}$ & 1 & $\frac{1}{2^{3}}$ & $\frac{13}{2^{8}}$ & $\frac{13}{2^{8}}$ & $\frac{13}{2^{8}}$ & $\frac{13}{2^{8}}$ & $\frac{1}{2^{2} \cdot 3^{2}}$ & $\frac{1}{2^{2} \cdot 3^{2}}$ & $\frac{1}{2^{2}}$ & $\frac{1}{2^{2}}$ & $\frac{1}{2^{3}}$ & $\frac{1}{2^{6}}$ & $\frac{1}{2^{6}}$ \\
$u_{i}$ & $\frac{1}{2^{2} \cdot 3^{2}}$ & $\frac{1}{2^{2}}$ & $\frac{1}{2^{2} \cdot 3^{2}}$ & $\frac{1}{2^{2} \cdot 3^{2}}$ & $\frac{1}{2^{2}}$ & $\frac{1}{2^{2}}$ & $\frac{2^{3}}{5}$ & $\frac{2^{3} \cdot 17}{3^{4} \cdot 5}$ & $\frac{2^{3} \cdot 17}{3^{4} \cdot 5}$ & $\frac{2^{3} \cdot 17}{3^{4} \cdot 5}$ & $\frac{1}{3^{2}}$ & $\frac{1}{3^{2}}$ & $\frac{1}{3^{2}}$ \\
$a_{j}$ & $\frac{1}{2^{3}}$ & $\frac{1}{2^{3}}$ & $\frac{1}{2^{6}}$ & $\frac{1}{2^{6}}$ & $\frac{1}{2^{6}}$ & $\frac{1}{2^{6}}$ & $\frac{1}{3^{2}}$ & $\frac{1}{3^{2}}$ & $\frac{1}{3^{2}}$ & $\frac{1}{3^{2}}$ & 1 & $\frac{1}{2^{3}}$ & $\frac{1}{2^{3}}$ \\
\hline
\end{tabular}

\subsection{Shape $(2 B, 3 C)$}

In the considered situation $T$ is the set of six $S_{4}$-transpositions. By Lemma 3.2, the algebra product is closed on the linear span of $\psi(T)$ :

$$
a_{(i j)} \cdot a_{(i j)}=a_{(i j)} ; \quad a_{(i j)} \cdot a_{(k l)}=0 ; \quad a_{(i j)} \cdot a_{(i k)}=\frac{1}{2^{6}}\left(a_{(i j)}+a_{(i k)}-a_{(j k)}\right) .
$$

The Gram matrix of $\psi(T)$ is non-singular, so the representation is 6-dimensional. The identity element is

$$
\iota=\frac{16}{17} \sum_{t \in T} a_{t} \text { and }(\iota, \iota)=\frac{2^{5} \cdot 3}{17} .
$$

\subsection{Shape $(2 A, 3 C)$}

Here $T$ is the set of all nine $S_{4}$-involutions and, as in the previous subsection, Lemma 3.2 applies:

$$
\begin{gathered}
a_{(i j)} \cdot a_{(i j)}=a_{(i j)} ; a_{(i j)} \cdot a_{(k l)}=\frac{1}{2^{3}}\left(a_{(i j)}+a_{(k l)}-a_{(i j)(k l)}\right) ; \\
a_{(i j)} \cdot a_{(i k)}=\frac{1}{2^{6}}\left(a_{(i j)}+a_{(i k)}-a_{(j k)}\right) ; a_{(i j)} \cdot a_{(i j)(k l)}=\frac{1}{2^{3}}\left(a_{(i j)}+a_{(i j)(k l)}-a_{(k l)}\right) ;
\end{gathered}
$$




$$
a_{(i j)} \cdot a_{(i k)(j l)}=\frac{1}{2^{6}}\left(a_{(i j)}+a_{(i k)(j l)}-a_{(k l)}-a_{(i l)(j k)}+a_{(i j)(k l)}\right) .
$$

The representation is 9-dimensional with identity element

$$
\iota=\frac{16}{21} \sum_{t \in T \backslash A_{4}} a_{t}+\frac{64}{105} \sum_{t \in T \cap A_{4}} a_{t}, \text { and }(\iota, \iota)=\frac{2^{5} \cdot 137}{3 \cdot 5 \cdot 7^{2}} \text {. }
$$

\section{Norton basis for $\mathcal{S}_{(2 B, 3 A)}$}

In this section $\zeta$ denotes the monomorphism $\zeta: S_{4} \rightarrow M$ such that $\zeta((i j)(k l))$ and $\zeta((i j k))$ belong to the conjugacy classes $2 B$ and $3 A$ of the Monster group $M$, respectively. Then $\mathcal{S}_{(2 B, 3 A)}$ is the subalgebra in the 196,884 dimensional Conway-Griess-Norton algebra generated by the Majorana axial vectors $a_{\zeta(t)}$ taken for all transpositions $t$ of $S_{4}$. Let $V_{M}$ denote the vector space underlying the Monster algebra. Although $\mathcal{S}_{(2 B, 3 A)}$ does not contain subalgebras of $4 A$-type, the image of $\zeta$ contains three cyclic subgroups generated by $4 A$-elements. It was suggested by Simon Norton that $\mathcal{S}_{(2 B, 3 A)}$ might contain $v_{\zeta(\rho)}$, where $\rho$ is an element of order 4 in $S_{4}$. To simplify notation, if $\rho$ is an element of order 4 in $S_{4}$ and $\rho^{2}$ maps $i$ onto $x$ then the vector $v_{\zeta(\rho)}$ will be denoted by $v_{x}$. The vector $v_{j}$ is invariant under $\zeta\left(N_{S_{4}}(\langle(i k j l)\rangle)\right) \cong D_{8}$. In order to find the candidates for $v_{j}$ in $\mathcal{S}_{(2 B, 3 A)}$, we have classified all idempotents $\vartheta$ in $\mathcal{S}_{(2 B, 3 A)}$ that are invariant under the subgroup

$$
D_{8}=\zeta(\langle(i k)(j l),(i j)\rangle)=\zeta\left(N_{S_{4}}(\langle(i k j l)\rangle)\right) .
$$

These idempotents might be of independent interest and are given in Table 10. The entries of the leading five columns show the coefficients of the relevant idempotent $\vartheta$ in the basis whose members are given in the headings of these columns, where

$$
a=a_{(i k)}+a_{(j k)}+a_{(i l)}+a_{(j l)}, \quad \sigma=\sigma_{i}+\sigma_{j}+\sigma_{k}+\sigma_{l} .
$$

The last two columns are reserved for inner products: by Table 3 , the scalar square of $v_{j}$ must be 2 and from the standard description of the Monster algebra in terms of the Leech lattice one can deduce that $\left(v_{j}, a_{(i j)}\right)$ must be $\frac{1}{24}$. 
Table 10

\begin{tabular}{|ccccc|cc|}
\hline$a_{(i j)}+a_{(k l)}$ & $a$ & $\sigma$ & $\delta_{(i j)(k l)}$ & $\delta_{(i k)(j l)}+\delta_{(i l)(j k)}$ & $(\vartheta, \vartheta)$ & $\left(\vartheta, a_{(i j)}\right)$ \\
\hline$\frac{m^{2}+15}{m^{2}+207}$ & $\frac{m+87}{m^{2}+207}$ & $\frac{64 m-1088}{m^{2}+207}$ & $\frac{4096 m-4096}{m^{2}+207}$ & $\frac{32768}{m^{2}+207}$ & 2 & $\frac{m^{2}}{m^{2}+207}$ \\
$\frac{1}{117}$ & $\frac{5}{6}$ & $-\frac{1216}{351}$ & $\frac{16384}{351}$ & $-\frac{2048}{27}$ & $\frac{46}{13}$ & $\frac{23}{624}$ \\
$\frac{58}{575}$ & $\frac{433}{575}$ & $-\frac{704}{115}$ & $\frac{53248}{575}$ & $-\frac{49152}{575}$ & $\frac{88}{25}$ & 0 \\
$\frac{31}{117}$ & $\frac{17}{26}$ & $-\frac{2944}{351}$ & $\frac{4096}{27}$ & $-\frac{2048}{27}$ & $\frac{46}{13}$ & $\frac{23}{624}$ \\
$\frac{344}{975}$ & $\frac{344}{975}$ & $\frac{1024}{585}$ & $\frac{16384}{225}$ & $\frac{16384}{225}$ & $\frac{644}{325}$ & $\frac{23}{52}$ \\
$\frac{22}{39}$ & 1 & $-\frac{320}{39}$ & $-\frac{4096}{39}$ & 0 & $\frac{72}{13}$ & $\frac{29}{52}$ \\
$\frac{88}{75}$ & $\frac{88}{75}$ & $-\frac{512}{45}$ & $\frac{16384}{225}$ & $\frac{16384}{225}$ & $\frac{188}{25}$ & 1 \\
\hline
\end{tabular}

Table 10 contains only half of the idempotents in question. The other half can be obtained by the following rule. The vector $\iota$ at the very bottom of the table is the identity of $\mathcal{S}_{(2 B, 3 A)}$, and for each $D_{8}$-invariant idempotent $\vartheta$ the vector $\iota-\vartheta$ is also an invariant idempotent with scalar square $\frac{188}{25}-(\vartheta, \vartheta)$. Otherwise the list is complete (provided that $m$ runs through the ground field and " $m=\infty$ ": the latter one gives the idempotent with coefficients $(1,0,0,0,0))$.

The vectors in the first row corresponding to $m= \pm 3$ satisfy all the requirements, although we could not conclude with full confidence that either of them is indeed the vector $v_{j}$ we are after. The reason is the following.

It was checked computationally by Steven Linton from St. Andrews that $\mathcal{S}_{(2 B, 3 A)}$ has codimension 1 in $X:=C_{V_{M}}\left(C_{(2 B, 3 A)}\right)$, where

$$
C_{(2 B, 3 A)}=C_{M}\left(\zeta\left(S_{4}\right)\right) \cong 2^{11} \cdot M_{23} .
$$

Because of the invariance, $v_{j}$ is definitely contained in $X$, but potentially it could be located outside $\mathcal{S}_{(2 B, 3 A)}$. This matter was settled by Simon Norton who has constructed an explicit embedding into the Monster algebra of the 7-dimensional subalgebra of $\mathcal{S}_{(2 B, 3 A)}$ formed by the vectors fixed by $\zeta((i j)(k l))$. It follows from his calculations that the vector $v_{j}$ is 
precisely the one corresponding to $m=-3$ in Table 10. Thus in Sakuma's basis we have

$$
\begin{array}{r}
v_{j}=\frac{1}{3^{2}}\left(a_{(i j)}+a_{(k l)}\right)+\frac{7}{2 \cdot 3^{2}}\left(a_{(i k)}+a_{(i l)}+a_{(j k)}+a_{(j l)}\right) \\
-\frac{2^{5} \cdot 5}{3^{3}}\left(\sigma_{i}+\sigma_{j}+\sigma_{k}+\sigma_{l}\right)-\frac{2^{11}}{3^{3}} \delta_{(i j)(k l)}+\frac{2^{12}}{3^{3}}\left(\delta_{(i k)(j l)}+\delta_{(i l)(j k)}\right) .
\end{array}
$$

The product rules of the algebra $\mathcal{S}_{(2 B, 3 A)}$ in the Norton basis

$$
a_{(i j)}, a_{(k l)}, a_{(i k)}, a_{(i l)}, a_{(j k)}, a_{(j l)}, u_{i}, u_{j}, u_{k}, u_{l}, v_{j}, v_{k}, v_{l}
$$

were calculated in [GAP4]:

$$
\begin{aligned}
& a_{(i j)} \cdot a_{(i j)}=a_{(i j)} ; \quad a_{(i j)} \cdot a_{(k l)}=0 ; \quad a_{(i j)} \cdot a_{(i k)}=\frac{1}{2^{5}}\left(2 a_{(i j)}+2 a_{(i k)}+a_{(j k)}\right)-\frac{3^{3} \cdot 5}{2^{11}} u_{l} ; \\
& a_{(i j)} \cdot u_{l}=\frac{1}{3^{2}}\left(2 a_{(i j)}-a_{(i k)}-a_{(j k)}\right)+\frac{5}{2^{5}} u_{l} \\
& a_{(i j)} \cdot u_{i}=\frac{1}{3^{3} \cdot 5}\left(11 a_{(i j)}-a_{(k l)}\right)+\frac{1}{2 \cdot 3^{3} \cdot 5}\left(a_{(i k)}+a_{(i l)}+a_{(j k)}+a_{(j l)}\right) \\
& +\frac{1}{2^{6} \cdot 3}\left(11 u_{i}+5 u_{j}-u_{k}-u_{l}\right)+\frac{1}{3^{2} \cdot 5}\left(v_{j}-2 v_{k}-2 v_{l}\right) \text {; } \\
& a_{(i j)} \cdot v_{j}=\frac{1}{2^{4} \cdot 3^{2}}\left(5 a_{(i j)}-a_{(k l)}\right)-\frac{1}{2^{2} \cdot 3^{2}}\left(a_{(i k)}+a_{(i l)}+a_{(j k)}+a_{(j l)}\right) \\
& +\frac{5}{2^{7}}\left(u_{i}+u_{j}+u_{k}+u_{l}\right)+\frac{1}{2^{4} \cdot 3}\left(v_{j}-2 v_{k}-2 v_{l}\right) ; \\
& a_{(i j)} \cdot v_{k}=\frac{1}{2^{6} \cdot 3^{2}}\left(85 a_{(i j)}+7 a_{(k l)}\right)-\frac{1}{2^{5} \cdot 3^{2}}\left(a_{(i k)}+a_{(i l)}+a_{(j k)}+a_{(j l)}\right) \\
& -\frac{1}{2^{10}}\left(70 u_{i}+70 u_{j}-5 u_{k}-5 u_{l}\right)-\frac{1}{2^{6} \cdot 3}\left(7 v_{j}-17 v_{k}-11 v_{l}\right) \text {; } \\
& u_{i} \cdot u_{i}=u_{i} ; \\
& u_{i} \cdot u_{j}=\frac{2^{8}}{3^{5} \cdot 5^{2}}\left(a_{(i j)}+a_{(k l)}\right)-\frac{2^{7}}{3^{5} \cdot 5^{2}}\left(a_{(i k)}+a_{(i l)}+a_{(j k)}+a_{(j l)}\right) \\
& +\frac{7}{2 \cdot 3^{3} \cdot 5}\left(2 u_{i}+2 u_{j}-u_{k}-u_{l}\right)+\frac{2^{6}}{3^{4} \cdot 5^{2}}\left(2 v_{j}-v_{k}-v_{l}\right) \text {; } \\
& u_{i} \cdot v_{j}=\frac{1}{3^{3} \cdot 5}\left(6 a_{(i j)}-2 a_{(k l)}-13 a_{(i k)}-13 a_{(i l)}+a_{(j k)}+a_{(j l)}\right) \\
& +\frac{1}{2^{6} \cdot 3}\left(45 u_{i}+11 u_{j}-8 u_{k}-8 u_{l}\right)+\frac{1}{3^{2} \cdot 5}\left(9 v_{j}-2 v_{k}-2 v_{l}\right) ;
\end{aligned}
$$




$$
\begin{gathered}
v_{j} \cdot v_{j}=v_{j} \\
v_{j} \cdot v_{k}=-\frac{5}{2^{2} \cdot 3^{3}}\left(a_{(i j)}+a_{(k l)}+a_{(i k)}+a_{(j l)}-2 a_{(i l)}-2 a_{(j k)}\right) \\
-\frac{5^{3}}{2^{9} \cdot 3}\left(u_{i}+u_{j}+u_{k}+u_{l}\right)+\frac{1}{2^{3} \cdot 3^{2}}\left(19 v_{j}+19 v_{k}+v_{l}\right) .
\end{gathered}
$$

The reduced Gram matrix in the Norton basis is given in Table 11. Notice that the disturbing large primes from the similar matrix in the Sakuma basis have disappeared. The determinant of the Gram matrix is

$$
\frac{2^{2} \cdot 11^{2} \cdot 23^{9}}{3^{20} \cdot 5^{6}} \text {. }
$$

Table 11

\begin{tabular}{|c|ccccccccccccc|}
\hline$()$, & $a_{(i j)}$ & $a_{(k l)}$ & $a_{(i k)}$ & $a_{(i l)}$ & $a_{(j k)}$ & $a_{(j l)}$ & $u_{i}$ & $u_{j}$ & $u_{k}$ & $u_{l}$ & $v_{k}$ & $v_{j}$ & $v_{l}$ \\
\hline$a_{(i j)}$ & 1 & 0 & $\frac{13}{2^{8}}$ & $\frac{13}{2^{8}}$ & $\frac{13}{2^{8}}$ & $\frac{13}{2^{8}}$ & $\frac{13}{2^{2} \cdot 3^{2} \cdot 5}$ & $\frac{13}{2^{2} \cdot 3^{2} \cdot 5}$ & $\frac{1}{2^{2}}$ & $\frac{1}{2^{2}}$ & $\frac{1}{2^{3} \cdot 3}$ & $\frac{31}{2^{6} \cdot 3}$ & $\frac{31}{2^{6} \cdot 3}$ \\
$u_{i}$ & $\frac{13}{2^{2} \cdot 3^{2} \cdot 5}$ & $\frac{1}{2^{2}}$ & $\frac{13}{2^{2} \cdot 3^{2} \cdot 5}$ & $\frac{13}{2^{2} \cdot 3^{2} \cdot 5}$ & $\frac{1}{2^{2}}$ & $\frac{1}{2^{2}}$ & $\frac{2^{3}}{5}$ & $\frac{2^{3} \cdot 7}{3^{3} \cdot 5^{2}}$ & $\frac{2^{3} \cdot 7}{3^{3} \cdot 5^{2}}$ & $\frac{2^{3} \cdot 7}{3^{3} \cdot 5^{2}}$ & $\frac{11}{3^{3}}$ & $\frac{11}{3^{3}}$ & $\frac{11}{3^{3}}$ \\
$v_{j}$ & $\frac{1}{2^{3} \cdot 3}$ & $\frac{1}{2^{3} \cdot 3}$ & $\frac{31}{2^{6} \cdot 3}$ & $\frac{31}{2^{6} \cdot 3}$ & $\frac{31}{2^{6} \cdot 3}$ & $\frac{31}{2^{6} \cdot 3}$ & $\frac{11}{3^{3}}$ & $\frac{11}{3^{3}}$ & $\frac{11}{3^{3}}$ & $\frac{11}{3^{3}}$ & 2 & $\frac{3^{2}}{2^{4}}$ & $\frac{3^{2}}{2^{4}}$ \\
\hline
\end{tabular}

\section{References}

[A94] M. Aschbacher, Sporadic Groups, Cambridge Univ. Press, Cambridge, 1994.

[C84] J.H. Conway, A simple construction for the Fischer-Griess monster group, Invent. Math. 79 (1984), 513-540.

[ATLAS $] \quad$ J.H. Conway, R.T. Curtis, S.P. Norton, R.A. Parker and R.A. Wilson, Atlas of Finite Groups, Clarendon Press, Oxford, 1985.

[GAP4] The GAP Group, GAP - Groups, Algorithms, and Programming, Version 4.4.12; 2008. (http://www.gap-system.org)

[Iv09] A.A. Ivanov, The Monster Group and Majorana Involutions, Cambridge Univ. Press, Cambridge 2009.

[MN93] W. Meyer and W. Neutsch, Associative subalgabras of the Griess algebra, $J$. Algebra 158 (1993), 1-17. 
[Miy96] M. Miyamoto, Griess algebras and conformal vectors in vertex operator algebras, J. Algebra 179 (1996), 523-548.

[Miy03] M. Miyamoto, Vertex operator algebras generated by two conformal vectors whose $\tau$-involutions generate $S_{3}, J$. Algebra 268 (2003), 653-671.

[Miy04] M. Miyamoto, A new construction of the moonshine vertex operator algebra over the real number field, Ann. Math. 159 (2004), 535-596.

[N96] S.P. Norton, The Monster algebra: some new formulae, In: Moonshine, the Monster and Related Topics, Contemp. Math. 193, pp. 297-306, AMS, Providence, RI, 1996.

[N98] S.P. Norton, Anatomy of the Monster I, In: The Atlas of Finite Groups: Ten Years On, LMS Lect. Notes Ser. 249, pp. 198-214, Cambridge Univ. Press, Cambridge, 1998.

[Sak07] S. Sakuma, 6-Transposition property of $\tau$-involutions of Vertex Operator Algebras, International Math. Research Notes 2007 article rnm030, 19 pages. 